\title{
Arginine Methyltransferase 1 in the Nucleus Accumbens Regulates Behavioral Effects of Cocaine
}

\author{
Yan Li (李燕), ${ }^{1 \star}$ Ruiming Zhu (朱睿明), ${ }^{1 \star}$ Wenjing Wang (汪文静), ${ }^{2 \star}$ Dengqi Fu (付登琦), ${ }^{1}$ Jing Hou (侯静), ${ }^{1}$ \\ Sen Ji (纪森), ${ }^{1}$ Bo Chen (陈波), ${ }^{1}$ Zhengtao Hu (扈正桃), ${ }^{1}$ Xue Shao (邵雪), ${ }^{1}$ Xuri Yu (余旭日), ${ }^{2}$ Qian Zhao (赵倩), ${ }^{1}$ \\ Baolai Zhang (张宝来), ${ }^{1}$ Changman Du (杜长蔓), ${ }^{1}$ Qian Bu (卜迁), ${ }^{1}$ Chunyan Hu (胡春燕), ${ }^{1}$ Yun Tang (唐芸), ${ }^{2}$ \\ - Lei Zhong (钟磊), ${ }^{2}$ Shengyong Yang (杨胜勇), ${ }^{2}$ Yinglan Zhao (赵瀛兰), ${ }^{2}$ and Xiaobo Cen (岑小波) ${ }^{1}$ \\ ${ }^{1}$ National Chengdu Center for Safety Evaluation of Drugs, State Key Laboratory of Biotherapy/Collaborative Innovation Center of Biotherapy, West China \\ Hospital, West China Medical School, Sichuan University, Chengdu 610041, People's Republic of China, and 2State Key Laboratory of Biotherapy and \\ Cancer Center, West China Hospital, Sichuan University, and Collaborative Innovation Center for Biotherapy, Chengdu 610041, People's Republic of China
}

Recent evidence suggests that histone modifications play a role in the behavioral effects of cocaine in rodent models. Histone arginine is known to be methylated by protein arginine $N$-methyltransferases (PRMTs). Evidence shows that PRMT1 contributes to $>\mathbf{9 0} \%$ of cellular PRMT activity, which regulates histone $\mathrm{H} 4$ arginine 3 asymmetric dimethylation (H4R3me2a). Though histone arginine methylation represents a chemical modification that is relatively stable compared with other histone alterations, it is less well studied in the setting of addiction. Here, we demonstrate that repeated noncontingent cocaine injections increase PRMT1 activity in the nucleus accumbens (NAc) of C57BL/6 mice. We, subsequently, identify a selective inhibitor of PRMT1, SKLB-639, and show that systemic injections of the drug decrease cocaine-induced conditioned place preference to levels observed with genetic knockdown of PRMT1. NAc-specific downregulation of PRMT1 leads to hypomethylation of H4R3me2a, and hypoacetylation of histone $\mathrm{H} 3$ lysine 9 and 14 . We also found that H4R3me2a is upregulated in NAc after repeated cocaine administration, and that H4R3me2a upregulation in turn controls the expression of $C d k 5$ and CaMKII. Additionally, the suppression of PRMT1 in NAc with lentiviral-short hairpin PMRT1 decreases levels of CaMKII and Cdk5 in the cocaine-treated group, demonstrating that PRMT1 affects the ability of cocaine to induce CaMKII and Cdk5 in NAc. Notably, increased H4R3me2a by repeated cocaine injections is relatively long-lived, as increased expression was observed for up to $7 \mathrm{~d}$ after the last cocaine injection. These results show the role of PRMT1 in the behavioral effects of cocaine.

Key words: behavioral plasticity; cocaine; histone arginine methylation; PRMT inhibitor; protein arginine $N$-methyltransferase 1

Significance Statement

This work demonstrated that repeated cocaine injections led to an increase of protein arginine $N$-methyltransferase (PRMT1) in nucleus accumbens (NAc). We then identified a selective inhibitor of PRMT1 (SKLB-639), which inhibited cocaine-induced conditioned place preference (CPP). Additionally, genetic downregulation of PRMT1 in NAc also attenuated cocaine-caused CPP and locomotion activity, which was associated with decreased expression of histone $\mathrm{H} 4$ arginine 3 asymmetric demethylation (H4R3me2a) and hypoacetylation of histone $\mathrm{H} 3$ lysine 9 and 14 (acH3K9/K14). This study also showed that H4R3me2a controlled transcriptions of Cdk5 and CaMKII, and that PRMT1 negatively affected the ability of cocaine to induce CaMKII and Cdk5 in NAc. Notably, increased H4R3me2a by repeated cocaine injection was relatively long-lived as increased expression was observed up to $7 \mathrm{~d}$ after withdrawal from cocaine. Together, this study suggests that PRMT1 inhibition may serve as a potential therapeutic strategy for cocaine addiction.

\section{Introduction}

Recent evidence suggests that histone modifications play a role in the behavioral effects of cocaine in rodent models (Renthal et al.,

Received Jan. 19, 2015; revised July 14, 2015; accepted July 16, 2015.

Author contributions: Y.L. and X.C. designed research; Y.L., R.Z., W.W., D.F., S.J., X.S., X.Y., Q.Z., B.Z., C.D., Y.T.,

L.Z., and Y.Z. performed research; S.Y. contributed unpublished reagents/analytic tools; Y.L., J.H., B.C., Z.H., Q.B.,

C.H., and Y.Z. analyzed data; Y.L. and X.C. wrote the paper.
2007; LaPlant et al., 2010; Maze et al., 2010a). Evidence shows that repeated noncontingent cocaine injections alter epigenetic modifications in the nucleus accumbens (NAc), including histone lysine acetylation or methylation, and DNA methylation 
(Renthal et al., 2007; Deng et al., 2010; LaPlant et al., 2010; Maze et al., 2010a). Histone arginine methylation, like lysine methylation, is an important epigenetic marker that occurs in eukaryotes, and plays a critical role in the transcriptional regulation and structural remodeling of chromatin (Bedford and Clarke, 2009; Lee and Stallcup, 2009). Presently, the functional consequences of histone arginine dimethylation are much less well understood than those of other post-translational modifications. In contrast to lysine acetylation or methylation, dimethylarginine is a more stable epigenetic modification, which is currently believed to be irreversible and to permanently label modified proteins (Sarmento et al., 2004; Bedford and Clarke, 2009; Di Lorenzo and Bedford, 2011). Dimethylarginine labeling differs significantly from histone lysine acetylation and/or methylation. Prior studies of histone changes in addiction have, however, focused on reversible epigenetic modifications (Renthal et al., 2007; Maze et al., 2010a). Given the long-lasting nature of addiction, irreversible histone modification may play an important role in the behavioral effects of cocaine and other drugs of abuse.

Protein arginine $N$-methyltransferases (PRMTs) are evolutionarily conserved from yeast to human and catalyze the transfer of a methyl group from $S$-adenosylmethionine to target arginine residues (Cheng et al., 2004; Pal and Sif, 2007; Sakamaki et al., 2011). PRMTs have been identified and included at least nine members that are generally classified into two types. Type I consists of PRMT1, 2, 3, 4, 6, and 8, which catalyze asymmetric dimethylarginines; type II consists of PRMT5, 7, and 9, which catalyze symmetric dimethylarginines (Nicholson et al., 2009). Of note, PRMT 1 contributes to $>90 \%$ of cellular PRMT activity, and regulates histone $\mathrm{H} 4$ arginine 3 asymmetric demethylation (H4R3me2a) in particular.

Recent studies and functional analyses indicate that PRMT1 is highly regulated in the brain in response to external stimuli, and that it is likely essential to varied brain functions (Birkaya and Aletta, 2005; Miyata et al., 2008; Wang et al., 2012b). For example, knockdown of PRMT1 reduces H4R3me2a levels, while decreasing proliferation and enhancing apoptosis in cells derived from glioma lines (Wang et al., 2012b). Moreover, PRMT1 is activated by nerve growth factor and may contribute to the activation of specific plasticity-related signal transduction pathways (Birkaya and Aletta, 2005). The modulation of PRMT1 activity may thus serve as a potential therapeutic target for disorders characterized by altered neuronal plasticity.

Here, we report that repeated noncontingent cocaine injections increase PRMT1 expression and activity in the NAc. We also report the identification of a selective inhibitor of PRMT1, SKLB-639, and demonstrate its ability to suppress cocaineinduced conditioned place preference (CPP) through a reduction in PRMT1-mediated modification of H4R3me2a.

\section{Materials and Methods}

\section{Drugs}

Cocaine- $\mathrm{HCl}$ was purchased from the National Institute for the Control of Pharmaceutical and Biological Products. Cocaine was dissolved in $0.9 \%$ saline. $5^{\prime}$-methylthioadenosine (MTA; D5011) and AMI-1 (A9232) were purchased from Sigma-Aldrich. MTA and AMI-1 were dissolved in

\section{The authors declare no competing financial interests.}

Correspondence should be addressed to either Xiaobo Cen or Yinglan Zhao, National Chengdu Center for Safety Evaluation of Drugs, State Key Laboratory of Biotherapy, West China Hospital of Sichuan University, 28 Gaopeng Street, High Technological Development Zone, Chengdu 610041, People's Republic of China, E-mail: xbcen@scu.edu.cn or alancenxb@sina.com.

DOI:10.1523/JNEUROSCI.0246-15.2015

Copyright $\odot 2015$ the authors $\quad 0270-6474 / 15 / 3512891-13 \$ 15.00 / 0$ dimethylsulfoxide (DMSO) and were diluted to the desired concentration before use.

\section{Animals}

Animals used in this study were adult male C57BL/6 mice (8-12 weeks old; weight, 22-24 g). The mice were housed five per cage in clear plastic cages with wire grid lids in a colony with a $12 \mathrm{~h}$ light/dark cycle (lights on from 7:00 A.M. to 7:00 P.M.) at constant temperature. Access to food and water was unrestricted. The animals were acclimatized for $7 \mathrm{~d}$ before the experiment. All animal protocols in this study were performed in accordance with the guidelines established by the Association for Assessment and Accreditation of Laboratory Animal Care.

\section{Homology modeling and structure-based virtual screening}

No crystal structures of mouse PRMT1 (mPRMT1) and human PRMT1 (hPRMT1) have been solved so far. The highly conserved rat PRMT1 (rPRMT1) x-ray structure [Protein Data Bank (PDB) codes 1OR8, 1ORI, and $1 \mathrm{ORH}]$ is available, though this protein lacks an important helical segment near the binding pocket (residues $1-40$ ). Moreover, rattus norvegicus PRMT3 (rPRMT3; PDB code 1F3L) and hPRMT3 (PDB code 3SMQ) structures are established. Since sequence alignments show that mPRMT1 and hPRMT1 are exactly the same within the binding pocket, we built a homology model of hPRMT1 (Zhang and Cheng, 2003; Yan et al., 2014) to process the virtual screening (see Fig. 4B). PRMT1 has two binding sites, including an $S$-adenoslymethionine (SAM) cofactor binding site and a substrate binding pocket. We considered these two sites as active to virtually screen our SKLB database. All screens were performed on Discovery Studio version 3.1 (Accelrys).

\section{Synthesis of SKLB-639}

4-Aminobenzamidine dihydrochloride $(1.6 \mathrm{~g}, 7.69 \mathrm{mmol})$ and triethylamine $(1.8 \mathrm{ml}, 24.48 \mathrm{mmol})$ were dissolved in EtOH $(150 \mathrm{ml})$, and then 4,6-dichloro-5-nitropyrimidine $(0.5 \mathrm{~g}, 2.58 \mathrm{mmol})$ was added. The reaction mixture was heated at $50^{\circ} \mathrm{C}$ for $3 \mathrm{~h}$. After cooling, the yellow suspension was filtered, washed with ethanol, and air dried to give 4,4'-((5-nitropyrimidine-4,6-diyl)bis(azanediyl))dibenzimidamide dihydrochloride $(1.27 \mathrm{~g}, 60 \%)$ as a yellow solid. The compound was identified by using liquid chromatography-mass spectrometry (MS) on a Quantum triple-quadrupole mass spectrometer in positive-ion mode using selected reaction monitoring $[1 \mathrm{H}$ nuclear magnetic resonance $(400 \mathrm{MHz}, \mathrm{DMSO}-\mathrm{d} 6): \delta 10.84(\mathrm{~s}, 2 \mathrm{H}), 9.39(\mathrm{~s}$, $4 \mathrm{H}), 9.16(\mathrm{~s}, 4 \mathrm{H}), 8.30(\mathrm{~s}, 1 \mathrm{H}), 7.89(\mathrm{~s}, 8 \mathrm{H}) \mathrm{ppm}$; MS (electrospray ionisation, positive ion) mass/charge ratio, $392.05(\mathrm{M}+\mathrm{H})]$.

\section{HPLC}

HPLC spectra for SKLB-639 were acquired by using the Waters 2695 2998 system, with the UV detection wavelength set to $295 \mathrm{~nm}$. Samples were injected $(10 \mu \mathrm{l})$ onto a Phenomenex $4.6 \times 150 \mathrm{~mm}, 5 \mu \mathrm{m} \mathrm{C} 18$ column at a temperature of $30^{\circ} \mathrm{C}$. The mobile phase was methanol/water (70:30) with a flow rate of $1.0 \mathrm{ml} / \mathrm{min}$. The final purity of synthesized SKLB-639 was $>98 \%$.

\section{Construction of lentiviral vectors for expressing shRNA of PRMT1} PLLU2G-shRNA PRMT1 and PLLU2G-control virus plasmids were constructed for the production of lentiviruses expressing shRNA of PRMT1 and green fluorescent protein (GFP; LV-shPRMT1), as follows: oligo sequence from $5^{\prime}$ to $3^{\prime}$, shPRMT1-F: TTGTTCCAGTATCTC CGATTATCTCGAGATAATCGGAGATACTGGAACATTTTTC; shPR MT1-R, TCGAGAAAAATGTTCCAGTATCTCCGATTATCTCGAGA TAATCGGAGATACTGGAACAA); lentivirus-expressing GFP (LVGFP), oligo sequence from $5^{\prime}$ to $3^{\prime}$ : Negative-F, TGCAACAAGATGAAGAGCACCAACTCGAGTTGGTGCTCTTCATCTTGTTGTTTTTT C; and Negative-R, TCGAGAAAAAACAACAAGATGAAGAGCAC CAACTCGAGTTGGTGCTCTTCATCTTGTTGCA). These two vectors contained the enhanced GFP coding sequence, allowing the identification of infected cells. All vector insertions were confirmed by dideoxy sequencing. Recombinant lentiviruses were produced by transient transfection in Neuro-2a cells, using PLLU2G-shRNA PRMT1 (to yield LV-shPRMT1) and PLLU2G-control (to yield LV- 
GFP). Viral titers were determined by infection of $293 \mathrm{~T}$ cells and GFP visualization $\left(2 \times 10^{9} \mathrm{TU} / \mathrm{ml}\right)$. Aliquots were kept at $-80^{\circ} \mathrm{C}$.

\section{Surgery}

Intra-NAc lentivirus. Under general ketamine/xylazine anesthesia, mice were positioned in a small-animal stereotaxic instrument, and the cranial surface was exposed. Thirty-three gauge syringe needles were used to bilaterally infuse $0.5 \mu \mathrm{l}$ of virus into the NAc at a $10^{\circ}$ angle [anteroposterior (AP), +1.6; mediolateral (ML), \pm 1.5 ; dorsoventral (DV), -4.4$]$ at a rate of $0.1 \mu \mathrm{l} / \mathrm{min}$. Animals receiving lentivirus injections were allowed to recover for 1 week following surgery before beginning the placeconditioning procedure to cocaine.

Intra-NAc mouse cannulations. Mice were surgically implanted with a sterilized catheter (28 gauge stainless steel, $5.0 \mathrm{~mm}$ projection). Each catheter was positioned on the skull just on the NAc (AP, +1.6; $\mathrm{ML},+1.5 ; \mathrm{DV},-4.4)$. Mice were allowed $7 \mathrm{~d}$ to recover from surgery before the start of place conditioning.

Intravenous catheterization. Mice were anesthetized with ketamine/ xylazine and prepared with long-term catheters (internal diameter, 0.40 $\mathrm{mm}$; external diameter, $0.48 \mathrm{~mm}$ ), as modified from a previous study (Belin et al., 2008). Briefly, the catheter was inserted into the right jugular vein and passed subcutaneously over the right shoulder to exit dorsally between the scapulae. Following surgery, animals were given $7 \mathrm{~d}$ to recover from surgery before starting self-administrated behavioral tests. During the first $4 \mathrm{~d}$ after surgery, animals received daily antibiotic treatment (penicillin, 160,000 U/ml, i.m.; $0.1 \mathrm{ml} /$ mice), and catheters were flushed with $0.04-0.06 \mathrm{ml}$ of heparanized saline $(30 \mathrm{U} / \mathrm{ml} 0.9 \%$ sterile saline).

\section{Drug injections}

Short-term and repeated cocaine injections. Mice used for detecting PRMT $\mathrm{mRNA}$ and/or protein received repeated ( $7 \mathrm{~d}, 20 \mathrm{mg} / \mathrm{kg}$, i.p.; once daily) and short-term (for $6 \mathrm{~d}$, saline; $1 \mathrm{~d}, 20 \mathrm{mg} / \mathrm{kg}$, i.p.) cocaine injections in the home cage. Mice that received repeated cocaine injections were killed $24 \mathrm{~h}$ after the last cocaine injection, and mice that received short-term cocaine injections were killed $1 \mathrm{~h}$ after the last injection.

Cocaine withdrawal. Mice were injected with cocaine or saline $(20$ $\mathrm{mg} / \mathrm{kg}$, i.p.) once per day for $7 \mathrm{~d}$. After 1, 7, 14, 28, and 42 withdrawal days, the mice were quickly decapitated, and their brains were rapidly removed and processed for Western blotting.

Intra-NAc MTA, AMI-1, and SKLB-639. For experiments investigating the effects of PRMT1 inhibitors on cocaine CPP, mice were surgically implanted with sterilized guide cannulae in NAc. Intra-NAc injections of MTA (500 $\mu \mathrm{M}, 1 \mu \mathrm{l} /$ injection, $0.1 \mu \mathrm{l} / \mathrm{min})$, AMI- 1 ( $1 \mathrm{~mm}, 1 \mu \mathrm{l} /$ injection, $0.1 \mu \mathrm{l} / \mathrm{min})$, SKLB-639 ( $1 \mathrm{~mm}, 1 \mu \mathrm{l} /$ injection, $0.1 \mu \mathrm{l} / \mathrm{min})$, and vehicle (0.5\% DMSO, $1 \mu \mathrm{l} /$ injection, $0.1 \mu \mathrm{l} / \mathrm{min}$; Hevia et al., 2004) were administered $30 \mathrm{~min}$ before cocaine or saline administration.

\section{Cocaine self-administration}

Cocaine self-administration was performed as previously described (Noonan et al., 2008). Sterilized catheters were surgically inserted into the right jugular vein of mice. Mice had $2 \mathrm{~h}$ of access daily to cocaine $(0.75$ $\mathrm{mg} / \mathrm{kg} /$ infusion) under a fixed ratio-1 reinforcement schedule. For PRMT1 Western blotting and PCR, mice underwent $7 \mathrm{~d}$ of cocaine selfadministration; then, they were killed $24 \mathrm{~h}$ after the last infusion.

\section{Conditioned place preference}

A cocaine CPP test in mice was performed as previously described, with slight modification (Valjent et al., 2006). All mice were handled for 3 consecutive days for $5 \mathrm{~min}$ each day before the experiment (days 1-3), after which mice were baseline tested at day 4 , and subsequently conditioned during days 5-10. At day 11 (CPP test 1), time spent in each compartment was measured. Mice were killed within 30 min after CPP test 1 .

\section{Locomotor activity}

Locomotor activity sessions were conducted once daily. Each mouse was placed in a locomotor activity chamber followed by intraperitoneal injection of $20 \mathrm{mg} / \mathrm{kg}$ cocaine or saline, and locomotor activity was measured for $30 \mathrm{~min}$, as previously described (Pritchard et al., 2012). The chambers were black acrylic boxes $(40.64 \times 40.64 \times 31 \mathrm{~cm})$ that were equipped with a top unit including a camera. Automated tracking was performed using EthoVision version 7.0 software (Noldus Information Technology).

\section{Tissue isolation}

At the end of each testing session, mice were killed by rapid decapitation. The NAc, striatum, hippocampus, and prefrontal cortex were removed from the brain, snap frozen in liquid nitrogen, and stored at $-80^{\circ} \mathrm{C}$ until protein extracts were prepared. For RNA isolation, samples were stored in RNAlater solution (Beyotime Institute of Biotechnology) and stored at $-80^{\circ} \mathrm{C}$ until processed further.

\section{Measurement of nuclear and cytoplasmic PRMT1 activity}

Nuclear and cytoplasmic extracts of fresh mouse NAc were separated using a commercial kit according to the manufacturer instructions (Beyotime Institute of Biotechnology). PRMT1 activity was subsequently determined with a Chemiluminescent Assay Kit (52004L, BPS Bioscience).

\section{Chemiluminescent assay to establish $I C_{50}$ values}

Chemiluminescent Assay Kits [PRMT3 (52005L), PRMT4 (52041L), PRMT5 (52002L), PRMT6 (52046), and PRMT8 (52058), BPS Bioscience) were used to determine the $\mathrm{IC}_{50}$ of compounds. Briefly, enzymatic reactions were conducted at room temperature for $30 \mathrm{~min}$ in a $50 \mu \mathrm{l}$ mixture containing proper methyltransferase assay buffer, $0.2 \mu \mathrm{M}$ $S$-adenoslymethionine, $2.5 \mathrm{ng}$ of PRMT1 enzyme, and the test compound (concentration range, $0.005-100 \mu \mathrm{M}$ ). The plates precoated with histone substrate were then performed as introductions. Finally, the luminescence of each well was measured in a BioTek Synergy 2 microplate reader. The luminescence data were analyzed and compared. In the absence of the compound, the intensity ( $\mathrm{Ce}$, the intensity of the absence of the compound) in each dataset was defined as $100 \%$ activity. In the absence of enzyme, the intensity ( $C 0$, the intensity of the absence of the enzyme) in each dataset was defined as $0 \%$ activity. The percentage of activity in the presence of each compound was calculated according to the following equation: percentage of activity $=(C-C 0) /(C e-C 0)$, where $C$ is the luminescence in the presence of the compound. [percentage of inhibition $=100-$ (percentage of activity).] IC $_{50}$ for compounds was calculated by nonlinear regression analysis of sigmoidal dose-response curves. To determine the $\mathrm{IC}_{50}$ of SKLB-639 on additional PRMTs, varied enzyme concentrations were used for each reaction [PRMT3, 25 ng/reaction; PRMT4, 200 ng/reaction; PRMT5, 100 ng/reaction; PRMT6, 100 ng/reaction; PRMT8, 50 ng/reaction].

\section{Western blot}

The expression levels of PRMT1 (catalog \#ab70724, abcam), PRMT4 (catalog \#4438, CST), PRMT5 (catalog \#2252, CST), H4R3me2a (catalog \#39705, Active motif), H3R17me2a (catalog \#A2421, ABclonal Technology), H3R2me2a (catalog \#A3155, ABclonal Technology), H2AR3me2a (catalog \#ab21574, abcam), dimethyl (sym; catalog \#ICP0811, Immunechem), hypoacetylation of histone $\mathrm{H} 3$ lysine 9 and 14 (acH3K9/K14; catalog \#9677, CST), H3K9me2 (catalog \#AH438, Beyotime Institute of Biotechnology), H3K36me3 (catalog \#ab9050, abcam), and $\beta$-actin (catalog \#4967, CST) were examined by Western blot as we did previously (Li et al., 2012).

\section{$R N A$ isolation and quantitative reverse transcription-PCR detection}

Bilateral NAc samples from mice treated with the indicated regimen of cocaine were dissected, homogenized in TRIzol, and then processed according to the manufacturer instructions. RNA was purified with RNeasy Micro Columns, and spectroscopy confirmed that the RNA 260/280 ratio was $>1.8$. RNA was then reverse transcribed using a Bio-Rad iScript Kit. The cDNA was quantified by quantitative reverse transcription-PCR (qRT-PCR) using SsoAdvanced SYBR Green Supermix. Each reaction was run in triplicate and analyzed following the $\Delta \Delta \mathrm{Ct}$ method, as previously described. All primer sequences are listed in Tables 1 and 2.

\section{Immunohistochemistry}

Mice were sedated with a lethal dose of chloral hydrate and perfused with $1 \times \mathrm{PBS}$ followed by $4 \%$ paraformaldehyde (PFA). Brains were post-fixed 
Table 1. Comprehensive list of all primer sequences used

\begin{tabular}{ll}
\hline Mouse mRNA primers & Primer sequence $\left(5^{\prime}-3^{\prime}\right)$ \\
\hline PRMT1-F & ACCTGACCTTCACCTCCCC \\
PRMT1-R & CTGCTTCCAGTGTGGTACGG \\
PRMT2-F & GGCGAACTGCGATTTGACA \\
PRMT2-R & GAACCCGTGACCACATCTCC \\
PRMT3-F & CCAATGGACTCAGTGAAAGCG \\
PRMT3-R & GGAGCAGGTTCTGACATCTACG \\
PRMT4-F & GTGGTCATCCCTGGCAAAGT \\
PRMT4-R & TCATCAGTGAAGGGTGGGA \\
PRMT5-F & GAGAATGCCCGACTACACA \\
PRMT5-R & AGCGGTAATGACGTGATTAG \\
PRMT6-F & GTGGATGGGTACGGACTTC \\
PRMT6-R & CATAGTGCTGCTTCACCTGGC \\
PRMT7-F & AACCAGCCTGATGAGAACG \\
PRMT7-R & CTCACACAAAGGCAGACGCTAC \\
PRMT8-F & AACACCGTGATCTTGCCAG \\
PRMT8-R & GCAGGTCATGTCAAAGCCATAG \\
Cdk5-F & GAGAAGATTGGGAAGGCAC \\
Cdk5-R & GAGGGCTGACTTGGCACAC \\
CaMKII-F & CCTGAAGCCTGAGAATCTGTTG \\
CaMKII-R & CGGGTCCTTCCTCAGCACT \\
Actin-F & GAGACCTTCAACACCCCAGC \\
Actin-R & ATGTCACGCACGATTTCCC \\
GAPDH-F & CCACTCCTCCACCTTTGAC \\
GAPDH-R & ACCCTGTTGCTGTAGCCA \\
\hline
\end{tabular}

$F$, Forward; $R$, reverse.

Table 2. Locations of mouse ChIP primers

\begin{tabular}{lll}
\hline Location & Mouse ChIP primers & Primer sequence $\left(5^{\prime}-3^{\prime}\right)$ \\
\hline Promoter region & Cdk5-F & CCGACTGAGGAACAATGGC \\
Promoter region & Cdk5-R & CTGGGTACATTGGGTAGGGC \\
Nonpromoter region & Cdk5-F & GATATGGTGGTGGGGTGGC \\
Nonpromoter region & Cdk5-R & GCTGAGTGTGGAGACAGGACTG \\
Promoter region & CaMKII-F & GAAGTGCTGAGGAAGGACCC \\
Promoter region & CaMKII-R & GCAGGGAGTAGTGGGAAGT \\
Nonpromoter region & CaMKII-F & ACTCAACAAATCAAACGACACG \\
Nonpromoter region & CaMKII-R & AAGCCGAGAGGATAAAGCTGTC \\
\hline
\end{tabular}

$F$, Forward; $R$, reverse.

overnight in 4\% PFA then were cryoprotected overnight in 30\% sucrose in PBS. Brains were sliced on a microtome at $10 \mu \mathrm{m}$. The brain sections underwent antigen retrieval. The sections then blocked, washed, and incubated with anti-PRMT1 (1:200; catalog \#2449, CST) antibodies and DyLight 488 (green) or 594 (red) fluorescent-coupled secondary antibodies. Nuclear costaining was achieved by incubating sections in $1 \times$ PBS containing DAPI $(2 \mu \mathrm{g} / \mathrm{ml})$ for $10 \mathrm{~min}$. Finally, sections were mounted in an antifade solution. All sections were imaged using a fluorescence microscope. And DAPI-positive and PRMT1-positive cells were counted by ImageJ software.

\section{Chromatin immunoprecipitation}

Fresh NAc were formaldehyde cross-linked and prepared for chromatin immunoprecipitation (ChIP) as previously described (Maze et al., 2010b). Tissue sonication and chromatin shearing were performed according to the manufacturer instructions (\#EZ 17-409, Millipore). Primer sequences were listed in Tables 1 and 2.

\section{Statistical analysis}

Statistical significance was measured using an unpaired two-tailed Student's $t$ test when comparing two groups with SPSS Statistics 21 software. One-way ANOVA following a Tukey's post hoc test was used to determine significance for CPP, Western blotting, and mRNA analysis with more than two groups using SPSS Statistics 21 software. For locomotion activity, cocaine-induced nucleus and cytoplasm activity alterations, and cocaine-induced alterations of PRMT1 protein nucleus and cytoplasm, two-way ANOVAs followed by Bonferroni post-tests were performed using GraphPad Prism 5, as experiments contained multiple groups. All values included in the figure legends represented the mean $\pm \mathrm{SEM}\left({ }^{*} p<\right.$ $\left.0.05 ;{ }^{* *} p<0.01 ;{ }^{* * *} p<0.001\right)$.

\section{Results}

\section{Increased expression of PRMT1 in NAc by repeated} cocaine administration

As a first step to determine the role of PRMTs in cocaine effects, we used qRT-PCR to screen basal transcriptional levels of PRMT1 to PRMT8 as well as expression in the NAc of mice that received repeated $(7 \mathrm{~d}, 20 \mathrm{mg} / \mathrm{kg}$, i.p.; once daily) and short-term (6 d, saline; 1 d, 20 mg/kg, i.p.) injections. Because PRMT9 is not expressed in mice, we did not measure mRNA expression of this form. We observed that PRMT1 and PRMT8 had the highest expression in NAc, while PRMT6 had the lowest expression. The relative levels of the other PRMTs in this region were similar (Fig. $1 A)$. The expression of PRMT1 mRNA in NAc was increased $(\sim 50 \%)$ by repeated cocaine injections compared with that of saline-treated mice (Fig. $1 B ; t_{(8)}=2.630, p<0.05$ ). In comparison, other PRMTs did not show significant changes in this brain region, with the exception of PRMT6, which showed a $28 \%$ decrease in expression (Fig. $1 B ; t_{(8)}=2.447, p<0.05$ ). Although both PRMT1 and PRMT6 are type I PRMTs, PRMT1 is the predominant type I PRMT in mammalian cells, accounting for $>90 \%$ of cellular PRMT activity (Bedford and Clarke, 2009; Li et al., 2010). Thus, we focused on investigating the role of PRMT1 in regulating cocaine-induced CPP. Consistent with PCR results, protein levels of PRMT1 were also increased following repeated cocaine injections (Fig. $1 C, t_{(6)}=4.207,{ }^{* *} p<0.01$ ). To test whether PRMT1 could be induced in the short term, we examined PRMT1 mRNA expression $1 \mathrm{~h}$ after a single cocaine injection. In this case, we observed similar alterations with repeated noncontingent cocaine injections (Fig. $1 D, t_{(6)}=2.622,{ }^{*} p<$ $0.05)$. Moreover, to determine whether increased expression of PRMT1 in NAc was specific, we examined PRMT1 expression in striatum, prefrontal cortex, and hippocampus after repeated noncontingent cocaine injections. We found that none of these brain regions showed changes (data not shown). Finally, with regard to whether PRMT1 was regulated by cocaine selfadministration, a long-term volitional delivery of cocaine, we examined the expression of PRMT1 $24 \mathrm{~h}$ after cocaine selfadministration (Fig. 1E). Our results showed that PRMT1 expression was elevated at the transcriptional level as well as at translational level following cocaine self-administration (Fig. $1 F$, $t_{(8)}=2.677,{ }^{\star} p<0.05$; Fig. $\left.1 G, t_{(6)}=2.535,{ }^{\star} p<0.05\right)$. Together, these data demonstrate that noncontingent cocaine exposure and cocaine self-administration increased the expression of PRMT1 in NAc.

To determine whether cocaine-induced CPP is associated with an elevation in PRMT1 expression in NAc, a series of experiments was performed. First, we showed that mice that received cocaine injections spent more time in a cocaine-paired chamber compared with mice that received saline (Fig. $2 B, t_{(22)}=7.458$, $\left.{ }^{* * *} p<0.001\right)$. Subsequently, we examined PRMT1 mRNA expression from NAc samples subjected to cocaine $24 \mathrm{~h}$ after CPP test. As shown in Figure $2 C$, cocaine CPP $\left(t_{(8)}=3.916,{ }^{* *} p<\right.$ 0.001 ) elevated PRMT1 mRNA production in NAc. Consistently, PRMT1 protein expression in NAc was increased, as demonstrated by Western blot (Fig. $2 D, t_{(4)}=4.276,{ }^{\star} p<0.05$ ). Moreover, immunostaining revealed that PRMT1 was predominantly expressed in the nucleus (Fig. 2E), and cocaine CPP was associated with an increase in the number of PRMT1-positive cells in NAc of mice (Fig. $2 F, t_{(17)}=2.907,{ }^{\star} p<0.05$ ). To further assess 

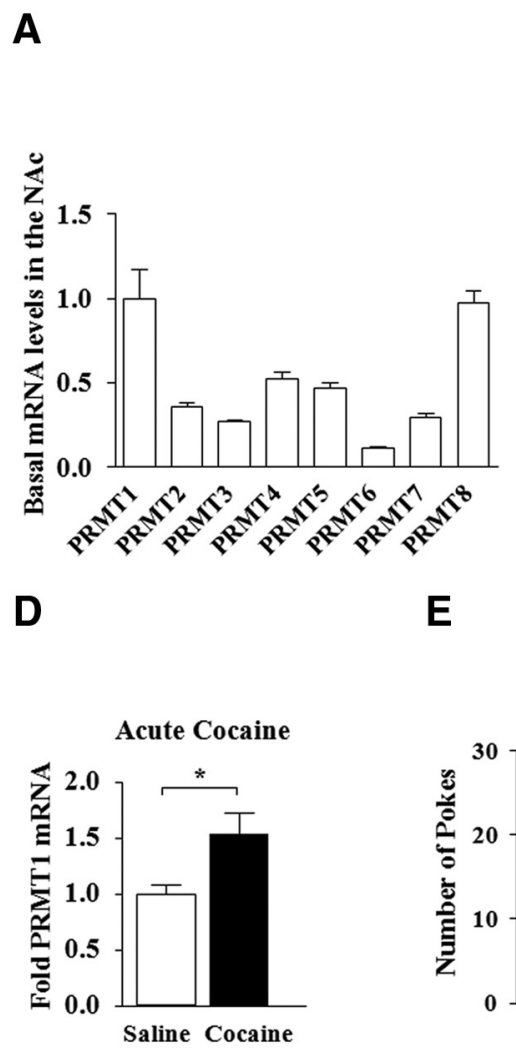

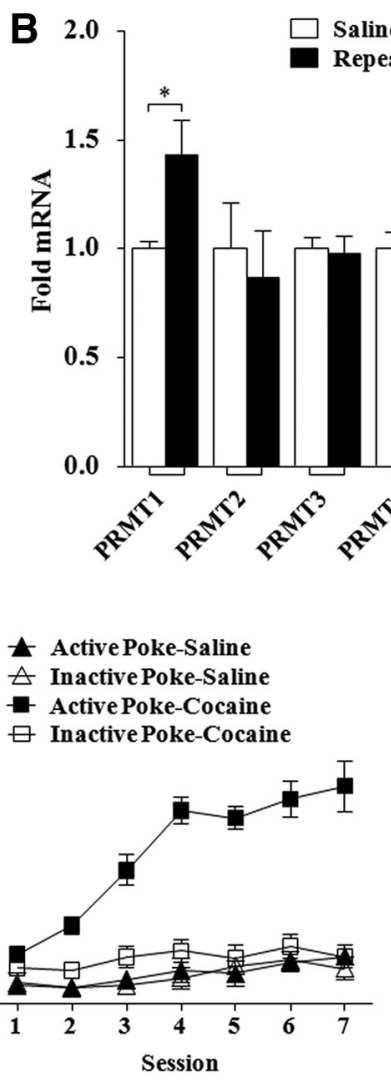

C

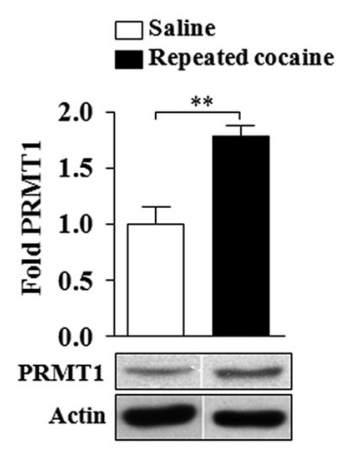

G

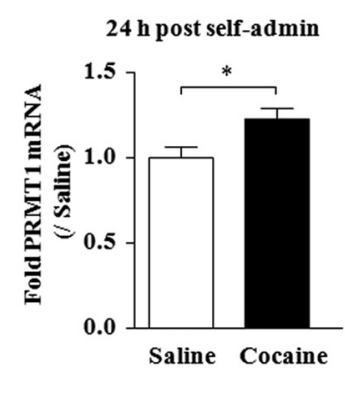

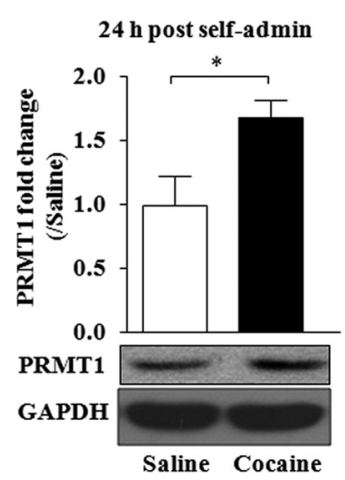

Figure 1. Regulation of PRMTs by cocaine administration (admin) in NAc. $A$, Basal expression levels of PRMT mRNA within the NAc expressed as the fold difference from PRMT1 from five mice. B, C57BL/6 mice were injected repeatedly ( $7 \mathrm{~d}, 20 \mathrm{mg} / \mathrm{kg}$, i.p., once daily) with cocaine. NAc samples were analyzed $24 \mathrm{~h}$ after the last injection. Student's $t$ tests, ${ }^{*} p<0.05, n=5$ saline, $n=5$ repeated cocaine. C, Repeated noncontingent cocaine injections upregulated PRMT1 protein expression in NAc (Student's $t$ tests, ${ }^{* *} p<0.01, n=4$ saline, $n=4$ repeated cocaine). D, Mice were injected over the short term with cocaine (6d, saline; $1 \mathrm{~d}, 20 \mathrm{mg} / \mathrm{kg}$, i.p.). NAc samples were analyzed $1 \mathrm{~h}$ after the last injection (Student's $t$ tests, ${ }^{*} p<0.05, n=4$ saline and $n=4$ acute cocaine). $\boldsymbol{E}$, The number of the "active" and "inactive" pokes for each daily session of cocaine self-administration, whereby active pokes resulted in cocaine infusion $(0.75 \mathrm{~g} / \mathrm{kg} / \mathrm{infusion})$ and the inactive pokes in both circumstances were without consequence. $n=9$ saline, $n=9$ cocaine. $F$, Cocaine self-administration caused elevated expression of PRMT1 mRNA in NAc. Student's $t$ tests, ${ }^{*} p<0.05, n=$ 5 saline, $n=5$ cocaine self-administration. G, Cocaine self-administration increased PRMT1 protein level. Actin was used as the loading control. Student's t tests, ${ }^{*} p<0.05, n=4$ saline, $n=4$ cocaine self-administration. Data are presented as the mean \pm SEM.

whether cocaine CPP redistributed PRMT1 across the nucleus and cytoplasm in NAc, we detected the PRMT1 activities both in the nuclear extract and cytoplasmic extract by using the Chemiluminescent Assay Kit (catalog \#52004L, BPS Bioscience). Results showed that cocaine CPP increased PRMT1 enzymatic activity by $46 \%$ (Fig. $2 G$; nuclear: $F_{(1,20)}=8.264,{ }^{* *} p<0.01$ ). However, this elevation was shown only in the nuclear extract, whereas no changes were observed in the cytoplasmic extract (Fig. 2G). Moreover, we detected PRMT1 protein levels in these subcellular fractions. We found that PRMT1 protein was increased both in the cytoplasm and nucleus in cocaine-treated mice; however, only nuclear PRMT1 was statistically significant $\left(\right.$ Fig. $2 H, t_{(7)}=$ $2.948,{ }^{*} p<0.01$ ), which was consistent with PRMT1 activity distribution.

\section{Development of selective bioactive PRMT1 inhibitor}

To study the role of PRMTs in cocaine reward, we tested the effect of intra-NAc MTA or AMI-1, nonspecific PRMT1 inhibitors (Cheng et al., 2004; Iwasaki and Yada, 2007; Limm et al., 2013). Based on previous studies, we used a 30 min time delay between inhibitors and cocaine injection (Hevia et al., 2004). We found that intra-NAc MTA or AMI- 1 attenuated cocaine CPP scores, as shown in Figure 3, $A$ and $B$, respectively. Importantly, the effect of AMI-1 on cocaine CPP scores was associated with reduced modification of H4R3me2a, which is asymmetrically methylated by PRMT1 (Fig. $3 C ; F_{(3,12)}=11.021,{ }^{\star} p<0.05$ ), whereas AMI-1 alone did not influence basal PRMT1 expression (Fig. 3D). To further investigate whether cocaine CPP specifically increased the histone mark of H4R3me2a, we studied the level of H2AR3me2a, another histone substrate of PRMT1. Results showed that H2AR3me2a modification was not affected by cocaine CPP (data not shown).

Because of the poor potency and selectivity of MTA and AMI-1 against PRMT1, we developed our own PRMT1 inhibitors with high potency and better selectivity. Similar to the discovery of other novel small-molecule inhibitors (Knutson et al., 2012; Lobera et al., 2013), a virtual screening against an in-house database $(15,039$ compounds) was first performed (see Materials and Methods). The first few hundred hits were clustered and visually inspected in consideration of the binding mode of known inhibitors of PRMT1 (Heinke et al., 2009; Bissinger et al., 2011; Cheng et al., 2011; Wang et al., 2012a). Initially, 19 compounds were tested using the Chemiluminescent Assay Kit (52004L, BPS Bioscience), and 4 of them showed inhibition of PRMT1. To get more potent and selective inhibitors, we synthesized and screened a small library of lead derivatives based on these four hits. Considering the known PRMT1 inhibitors, we first synthesized a lot of symmetric structural compounds with 5-nitropyrimidine-4,6-diamine. Among them, amidine moiety was 
A
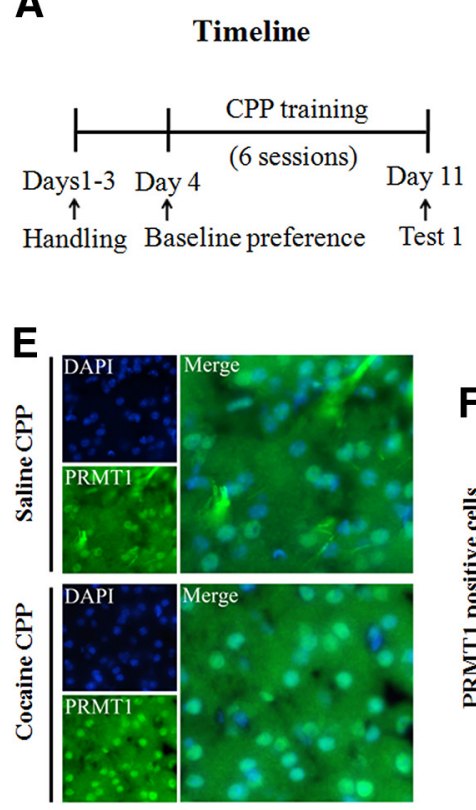

B

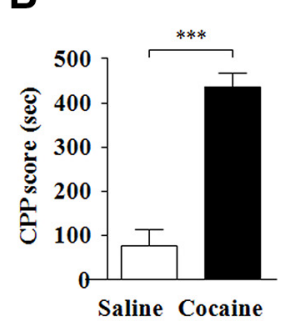

C

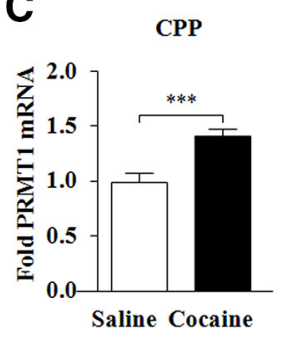

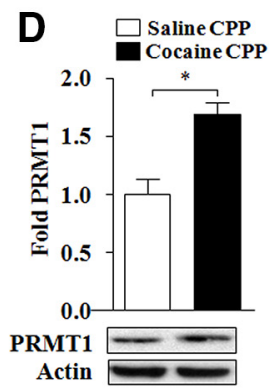

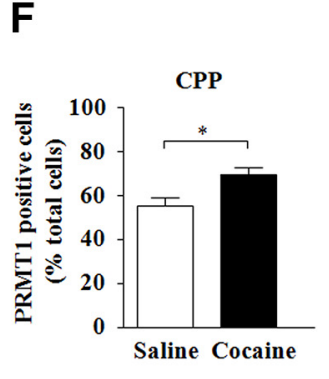

G

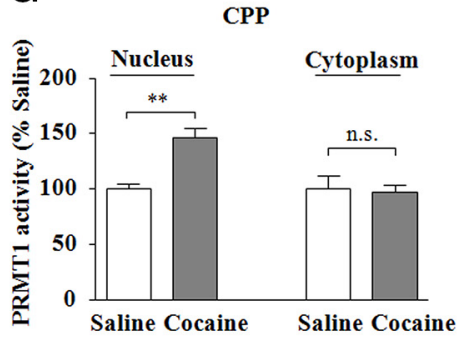

H

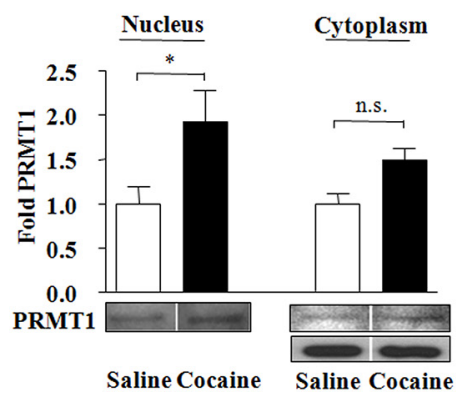

Figure 2. PRMT1 is increased following cocaine-induced conditioned place preference. $\boldsymbol{A}$, Timeline of cocaine CPP experiment. $\boldsymbol{B}$, Cocaine CPP. After conditioning, mice developed a significant preference for the cocaine-paired side. Student's $t$ tests, ${ }^{* * *} p<0.001, n=12$ saline, $n=12$ cocaine. C, Cocaine CPP caused significant elevation of PRMT1 expression in mRNA level. Student's $t$ tests, ${ }^{* * *} p<0.001, n=5$ saline, $n=5$ cocaine. $\boldsymbol{D}$, Representative immunoblot demonstrating elevated PRMT1 expression in NAc following cocaine CPP. Actin was used as the loading control. Student's $t$ tests, ${ }^{*} p<0.05, n=3$ saline, $n=3$ cocaine. $\boldsymbol{E}$, PRMT1 protein expression in NAc measured by immunohistochemistry, $24 \mathrm{~h}$ following cocaine (PP ( $40 \times$ objective). $\boldsymbol{F}$, Ratio of merged positive cells and DAPI-positive cells from $\boldsymbol{E}$ were counted and averaged, respectively. There were a total of $2348 \mathrm{DAPI}$-positive cells after saline CPP, which were counted from 8 photos in three mice, whereas 2696 cells were counted from 11 photos in four mice challenged by cocaine CPP. Student's $t$ tests, ${ }^{*} p<0.05$. G, PRMT1 activity was detected in nucleus and cytoplasm in NAc after cocaine CPP. The PRMT1 activity in nucleus was obviously increased. ${ }^{* *} p<0.01$. n.S., Not significant. $n=6$ saline, $n=6$ cocaine. Data are presented as the mean \pm SEM. $\boldsymbol{H}$, Levels of PRMT1 in cytoplasm and nuclear fractions in NAc from cocaine CPP mice were detected by Western blot. PRMT1 protein in nuclear extract was statistically increased. ${ }^{*} p<0.01$. n.s., not significant, $n=4$ saline, $n=4$ cocaine.

found to be very important to the activity of this series. Docking was performed to understand the binding mode of these compounds. We found that amidine moiety binds deeply into the substrate binding site, and forms hydrogen bond interactions with Glu161 and other amino acids, just like the arginine residue of the substrate. We then synthesized a series of asymmetric structural compounds with 5-nitropyrimidine-4,6-diamine as the scaffold and amidine as the tail. Finally, we identified a symmetric structural SKLB-639 (Fig. $4 A$ ), which effectively inhibited the methylation of H4R3me2a by PRMT1 with an $\mathrm{IC}_{50}$ value of $2.4 \mu \mathrm{M}$, which was more potent than the well known pan-PRMT inhibitor AMI-1 $\left(\mathrm{IC}_{50}=20 \mu \mathrm{M}\right)$ against PRMT1 in the same assay (Fig. 4E).

We further studied the differences in the mechanism of action and possible binding mode between SKLB-639 and PRMT1. Results showed that $\mathrm{IC}_{50}$ values of SKLB-639 increased linearly in the presence of increasing concentrations of $\mathrm{H} 4$ peptide concentrations. On the contrary, $\mathrm{IC}_{50}$ values of SKLB-639 remained constant with the concentration variation of the cofactor $S$-adenoslymethionine (data not shown). These results indicate that SKLB-639 is competitive with the substrate $\mathrm{H} 4$ peptide, whereas it is noncompetitive with the cofactor $S$-adenoslymethionine. Together, the above biochemical assays suggest that SKLB-639 binds in the substrate binding pocket of PRMT1, which confirmed our prior hypothesis.

Docking was then conducted to understand the possible binding mode and the detailed interactions of SKLB-639 with hPRMT1. Figure 4, $B$ and $C$, showed the predicted interaction mode of SKLB-639 with hPRMT1 in the substrate binding pocket. One amidine group of SKLB-639 occupied the substrate binding pocket, which was a narrow channel formed by side chains of Tyr47, Ile52, Met56, Trp302, and His301, and the back- bone of residues Glu152-Tyr156. The amidine group was engaged in extensive interactions with the protein, with this moiety forming electrostatic and hydrogen bond interactions with Glu161, which was the one of the conserved active sites, also known as Double-E. In addition, it also formed hydrogen bonds with residues Met154, Tyr47, and His301. Met154 was another important conserved residue in the PRMT substrate-binding site that interacts with arginine. The presence of the other amidine group was involved in hydrogen bonding to Arg361 and Tyr163, which explained the high activity of SKLB-639 over other asymmetric structure compounds, with 5-nitropyrimidine-4,6diamine as the scaffold. Additionally, the nitro-group also made a hydrogen bond with Tyr156 (Fig. 4D).

We next determined the selectivity of SKLB-639 for PRMT1 against other PRMT family members. PRMT3, 4, 5, 6, and 8 were chosen in this assay. We did not examine PRMT2 and PRMT7, because no activity was demonstrated for PRMT2, and poorly characterized activity was found for PRMT7 (Bedford and Clarke, 2009). Results showed that $\mathrm{IC}_{50}$ values of SKLB-639 against PRMT3 and PRMT4 were 36.4 and $73.9 \mu \mathrm{M}$ (Fig. $4 F, G$ ), indicating a selectivity of $>15$ - and 30 -fold, respectively. Notably, the $\mathrm{IC}_{50}$ values of SKLB-639 against PRMT5, 6, and 8 were all $>100 \mu \mathrm{M}$ (Fig. $4 F, J$ ). All of these data indicate that SKLB-639 is a potent and selective inhibitor of PRMT1.

\section{SKLB-639 suppresses cocaine-induced behavior}

We next assessed the behavioral actions of SKLB-639 in the cocaine CPP paradigm following intra-NAc delivery (Fig. 5A). Notably, SKLB-639 caused a decrease in CPP scores (Fig. 5B, $\left.F_{(3,43)}=13.878,{ }^{*} p<0.05,{ }^{* *} p<0.001\right)$. Subsequently, we 
examined the protein levels of H4R3me2a in NAc samples from cocaine CPPexperienced mice. The modification of H4R3me2a was increased after treatment with vehicle plus cocaine, while its expression was not changed in an SKLB-639 plus cocaine challenge, compared with a vehicle plus saline injection (Fig. 5C). Additionally, in this assay, SKLB-639 alone caused an $\sim 0.2$-fold decrease in H4R3me2a within NAc (Fig. 5C). However, consistent with in vitro results, SKLB-639 did not affect the expression of H3R2me2a and H3R17me2a modifications that followed from the activity of PRMT6 and PRMT4 (Fig. 5D,E), respectively (Guccione et al., 2007; Selvi et al., 2010), nor did it affect the dimethyl (sym) mediated by type II PRMTs (Nicholson et al., 2009; Fig. 5F). Moreover, we also found that cocaine alone did not influence the expression of H3R2me2a, H3R17me2a, and dimethyl (sym). Combined with our in vitro results, these data suggest that the biological activity of NAc PRMT1, in particular, plays an important role in the behavioral actions of cocaine.

\section{Downregulation of PRMT1 in NAc modulates behavioral responses to cocaine}

From the studies described above, we observed that the inhibition of PRMT1 enzymatic activity by SKLB-639 attenuated cocaine CPP. To further test whether genetic downregulation of PRMT1 can influence cocaine-induced rewards, we constructed a lentivirus to specifically express shRNA of PRMT1 in NAc. We first designed three types of shRNA targeting different PRMT1 isoform-specific sequences and without any binding sites to other PRMTs. We selected the vector that showed the most effective inhibition of PRMT1 expression, but without effects on the expression of other PRMT isoforms. We next examined the effect of PRMT1 downregulation on cocaine-elicited behaviors by giving mice bilateral intra-NAc injections of vectors expressing either GFP (LV-GFP) or LV-shPRMT1

(Fig. 6A). Importantly, mice expressing PRMT1 shRNA in NAc displayed a reduced preference for cocaine in contrast to those expressing GFP (Fig. $6 B, F_{(3,45)}=22.036,{ }^{*} p<0.05,{ }^{* *} p<0.01$, ${ }^{* * *} p<0.001$ ), which is equivalent to the levels observed for pharmacological inhibition of PRMT1 (Fig. 5B). Furthermore, NAc-specific PRMT1 downregulation impaired cocaine-induced locomotor sensitization (Fig. $6 C, F_{(3,308)}=219.5,{ }^{\star} p<0.05$, $\left.{ }^{* *} p<0.01,{ }^{* *} p<0.001\right)$; however, neither the CPP score nor the basal locomotor activity was affected by LV-shPRMT1 alone (Fig. 6B,C). To verify that the attenuation of cocaine-induced behavioral plasticity was associated with PRMT1 disruption, qRT-PCR and Western blotting were used to detect PRMT1 ex-

C
Timeline

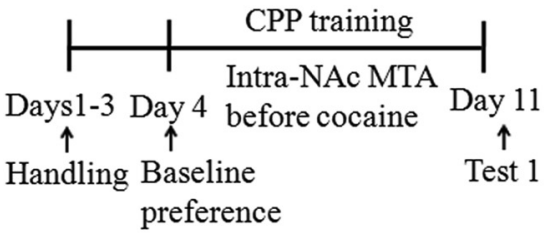

ntra-NAc MTA
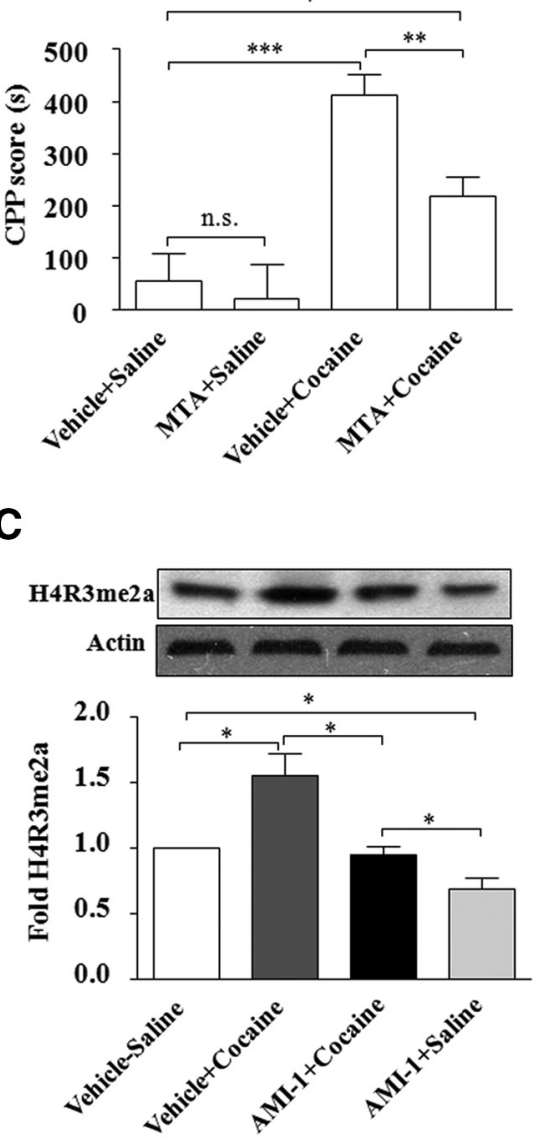

Figure 3. Intra-NAc injections of PRMT inhibitors regulate cocaine-induced behavioral plasticity. $A$, Intra-NAc MTA (500 $\mu \mathrm{M}, 1$ (attenuated cocaine CPP. ${ }^{*} p<0.05,{ }^{* *} p<0.01,{ }^{* * *} p<0.001 . n=9$ Vehicle +Saline, $n=8$ MTA + Saline, $n=$ 8 Vehicle + Cocaine, $n=11$ MTA + Cocaine. $\boldsymbol{B}$, Intra-NAc AMI-1 ( $1 \mathrm{~mm}, 1 \mu \mathrm{l} /$ injection) partially blocked cocaine CPP. ${ }^{*} p<0.05$ ${ }^{* * *} p<0.001 . n=13$ Vehicle + Saline, $n=9$ AMI-1 + Saline, $n=12$ Vehicle + Cocaine, $n=12$ AMI-1 + Cocaine. C, Intra-NAc $n=4$ AMI-1 + Saline, $n=4$ Vehicle + Cocaine, $n=4$ AMI-1+Cocaine. D, Intra-NAc AMI-1 (1 mM, $1 \mu \mathrm{l} /$ injection) did not influence basal PRMT1 expression in NAc. ${ }^{*} p<0.05$. n.s., Not significant. $n=4$ Vehicle + Saline, $n=4$ AMI- $1+$ Saline, $n=4$ Vehicle + Cocaine, $n=4$ AMI- $1+$ Cocaine. Data are presented as the mean \pm SEM. pression. As expected, the levels of PRMT1 mRNA as well as of protein in NAc after the cocaine CPP procedure were downregulated by LV-shPRMT1 cocaine when compared with LV-GFP cocaine (Fig. $6 D, F_{(3,20)}=15.615,{ }^{\star} p<0.05,{ }^{* *} p<0.01$; Fig. $6 E$, $\left.F_{(3,16)}=20.91,{ }^{\star} p<0.05,{ }^{* *} p<0.001\right)$. Immunostaining also revealed a weakened PRMT1 immunoreactivity in NAc of mice treated with LV-shPRMT1 (Fig. 6F).

We next examined the changes in H4R3me2a. As shown in Figure $6 G$, we observed that $\mathrm{H} 4 \mathrm{R} 3 \mathrm{me} 2 \mathrm{a}$ expression was increased in the LV-GFP cocaine group, but was inhibited in the LV-shPRMT1 cocaine group, which was coincident with PRMT1 reduction. Since published studies suggest that PRMT1 controls H4R3me2a and sub- 


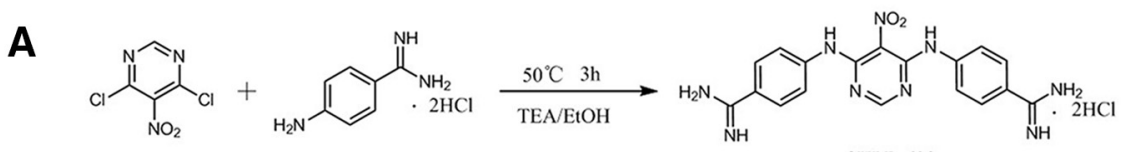

SKLB-639

B

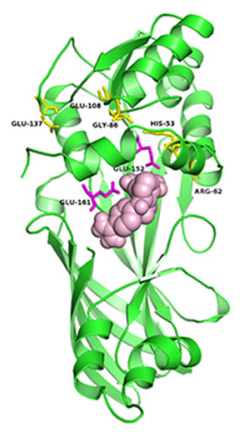

E

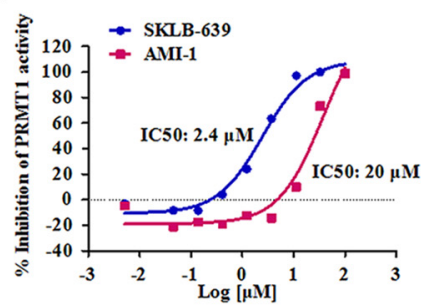

H

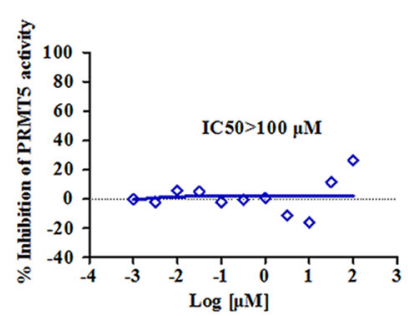

C

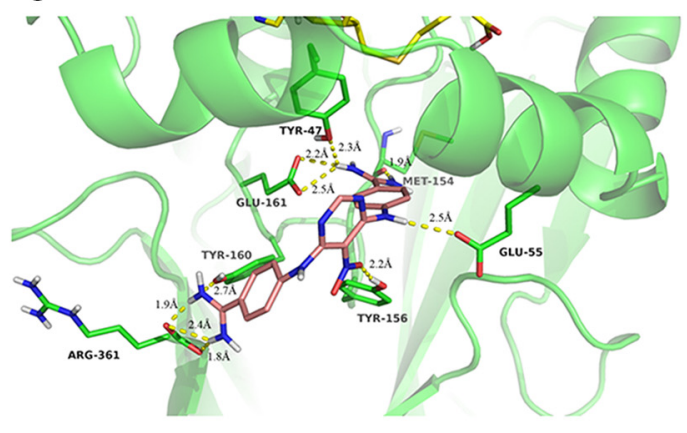

$F$

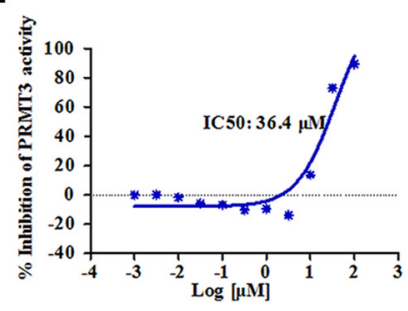

I

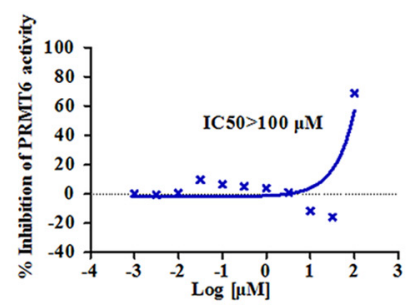

D

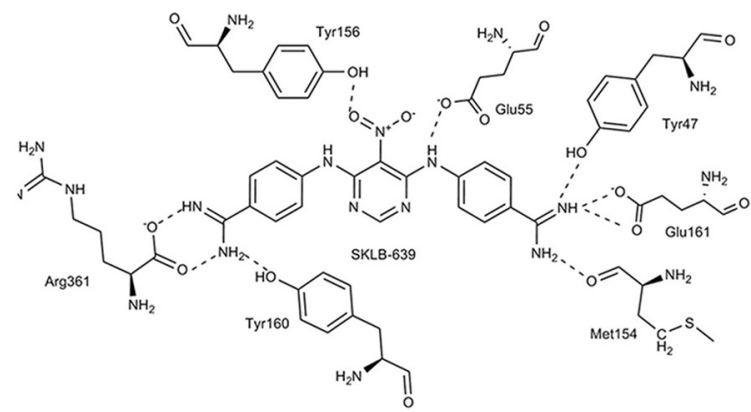

G

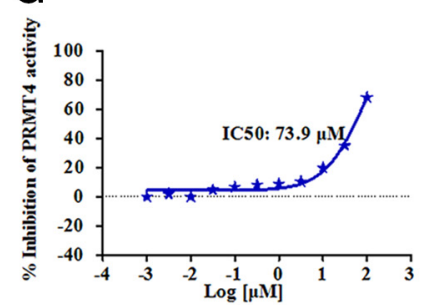

J

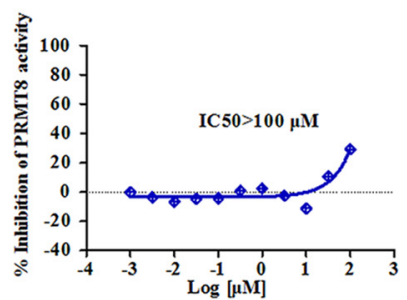

Figure 4. Discovery of a potent and selective inhibitor of PRMT1. A, Synthesis of the SKLB-639. B, The overall structure of homology hPRMT1 with SKLB-639 (light pink). Regions are colored as follows: SAM-binding site (yellow: residues His53, Arg62, Glu86, Glu 108, Glu 137); and active site (magenta: residues Glu152 and Glu161). C, Predicted binding modes of SKLB-639 in hPRMT1 homology model from docking and molecular dynamics simulation (Discovery Studio version 3.1). D, Substrate binding sites of SKLB-639 in PRMT1 were Glu161, Met154, Tyr47, His301, Arg361, and Tyr156. E, Concentration-response curve of SKLB-639 (blue) and AMI-1 (red) in the PRMT1 in vitro assay. F-J, Selectivity of SKLB-639 on five other protein arginine methyltransferases.

sequently affects histone acetylation at site $\mathrm{H} 3 \mathrm{~K} 9 / \mathrm{K} 14$ (Fig. $6 G$, $F_{(3,12)}=11.92,{ }^{\star} p<0.05,{ }^{* *} p<0.01$; Li et al., 2010). We also asked whether a loss of asymmetric dimethyl H4R3 resulted in the disruption of $\mathrm{H} 3 \mathrm{~K} 9 / \mathrm{K} 14$ acetylation modification in NAc in cocaine reward. We thus examined the changes in $\mathrm{H} 3 \mathrm{~K} 9 / \mathrm{K} 14$ acetylation after LV-shPRMT1 cocaine treatment. Strikingly, the expression of acH3K9/K14 was also decreased by LV-shPRMT1 compared with LV-GFP, supporting a model in which histone H4 Arg3 methylation is a critical requirement for subsequent histone $\mathrm{H} 3$ acetylation (Fig. $6 A, D$; Li et al., 2010). These results indicate that NAc-specific PRMT1 knockdown may serve to counter the rewarding response to the drug.

\section{H4R3me2a regulates $C d k 5$ and $C a M K I I$ gene expressions and is relatively longer lived when modified by repeated noncontingent cocaine injection}

Prior investigations have shown that histone lysine acetylation and methylation modifications play crucial roles in gene transcription. In particular, it has been shown that these changes control the transcriptional activation state at the promoter regions of $C d k 5$ and CaMKII (Kumar et al., 2005). Therefore, we hypothesize that alterations in $\mathrm{H} 4 \mathrm{R} 3 \mathrm{me} 2 \mathrm{a}$ and $\mathrm{acH} 3 \mathrm{~K} 9 /$ K14 by PRMT 1 in NAc might affect the expression of $C d k 5$ and CaMKII, both of which have previously been demonstrated to influence aspects of drug action (Bibb et al., 2001; Kumar et al., 2005; Kadivar et al., 2014). We thus tested the binding of $\mathrm{H} 4 \mathrm{R} 3 \mathrm{me} 2 \mathrm{a}$ and acH3K9/K14, respectively, to the promoters of these putative gene targets after repeated cocaine administration by using ChIP. We found that Cdk5 and CaMKII showed exclusively increased binding of H4R3me2a and acH3 K9/K14 after repeated cocaine injections, indicating potentially increased transcriptional activation of $C d k 5$ and CaMKII (Fig. 7 A, C).

To validate that alterations in H4R3me2a and acH3K9/K14 interactions with repeated cocaine challenge were associated with transcriptional changes, we measured the mRNA levels of CaMKII and Cdk5 by using qRT-PCR. We found that both of them displayed markedly increased mRNA expression after repeated noncontingent cocaine injections (Fig. $7 B, t_{(8)}=3.303,{ }^{*} p<$ 0.05; Fig. $\left.7 D, t_{(8)}=2.463,{ }^{\star} p<0.05\right)$. Additionally, we also found 
that, with the suppression of PRMT1 in NAc, the levels of CaMKII and Cdk5 in the LV-shPRMT1 cocaine group were decreased compared with that of the LVGFP cocaine group following use of the CPP apparatus (Fig. 7E, ${ }^{\star} p<0.05$ ). These results suggested that PRMT1 negatively affected the ability of cocaine to induce CaMKII and Cdk5 in NAc.

To examine the longevity of the increased modification of H4R3me2a by repeated noncontingent cocaine injections, we analyzed NAc samples at a global level at several time points during cocaineinduced withdrawal. We found that the level of H4R3me2a was elevated after $1 \mathrm{~d}$ of withdrawal, and that this significant elevation remained for $7 \mathrm{~d}$ (Fig. $7 F$ : day 1 , $t_{(4)}=6.906,{ }^{\star} p<0.05$; day $7, t_{(4)}=4.886$, $\left.{ }^{*} p<0.05\right)$. At $14 \mathrm{~d}$ after withdrawal, this effect was no longer statistically significant and was beginning to return to a normal level. To determine potentially longerlasting modifications of H4R3me2a, we detected the levels of H3K9me2 and $\mathrm{H} 3 \mathrm{~K} 36 \mathrm{me} 3$ at the same time points. In contrast to H4R3me2a, the levels of $\mathrm{H} 3 \mathrm{~K} 9 \mathrm{me} 2$ and H3K36me3 were decreased by repeated cocaine injections after $1 \mathrm{~d}$ of withdrawal and quickly returned to normal level (Fig. $7 G, t_{(4)}=4.010$; Fig. $\left.7 H, t_{(4)}=6.904\right)$. These results provide a novel chromatin-based mechanism that PRMT1 regulates cocaine-induced reward through modulating the histone methylation of arginine residues in a longterm manner, eventually regulating the transcriptional activation of target genes, which appears to be a key mechanism of cocaine-induced behavioral plasticity.

\section{Discussion}

Our results reveal a novel histone modification that occurs with cocaineinduced CPP. We describe the development of an inhibitor of PRMT1, SKLB-639, that, in contrast to prior compounds, is selective both in vitro and in vivo. NAc-targeted manipulations using small-molecule chemical inhibitors or PRMT1 knockdown to specifically inhibit PRMT1 activity attenuate cocaine-induced CPP in mice. We additionally demonstrate that, compared with histone lysine modification, H4R3me2a is a relatively long-lived change that occurs with repeated noncontingent cocaine exposure. Our findings reveal a new and effective pharmacological strategy to selectively inhibit PRMT1 activity, and we demonstrate a novel form of plasticity that occurs in the background of cocaine-associated plasticity.

Since the first protein methyltransferase was characterized, $>50$ human protein methyltransferases have been identified (Vedadi et al., 2011). Therefore, over the past decade, numerous
B
Timeline
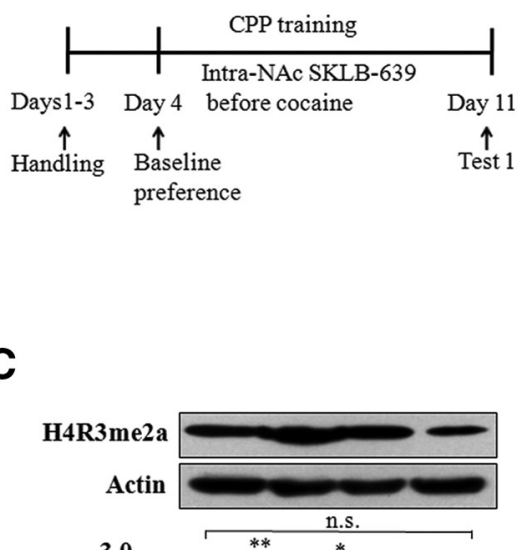

E

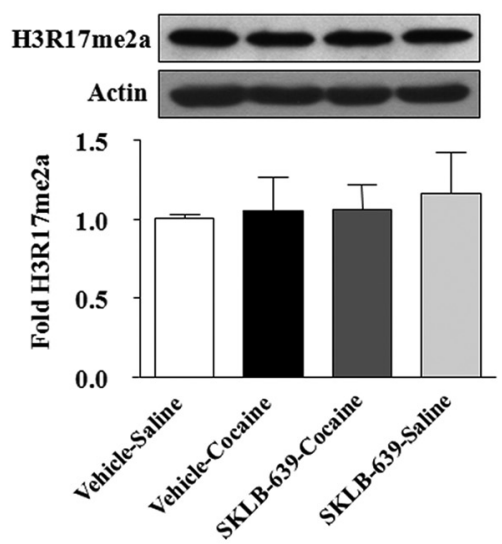

Intra-NAc SKLB-639

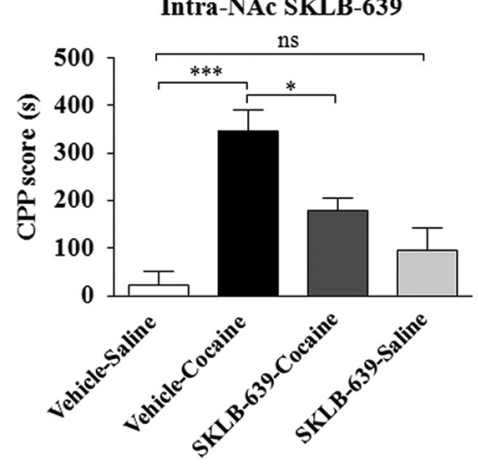

D
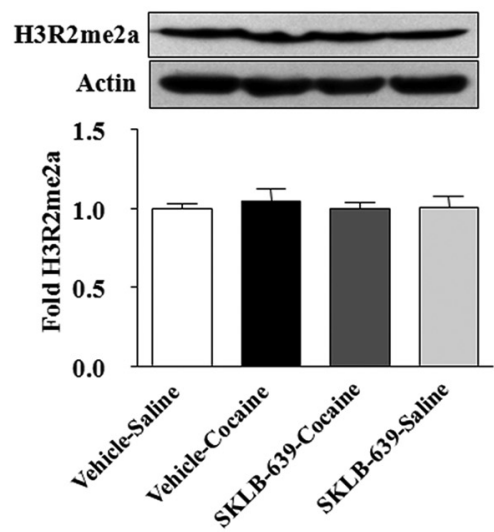

$\mathbf{F}$

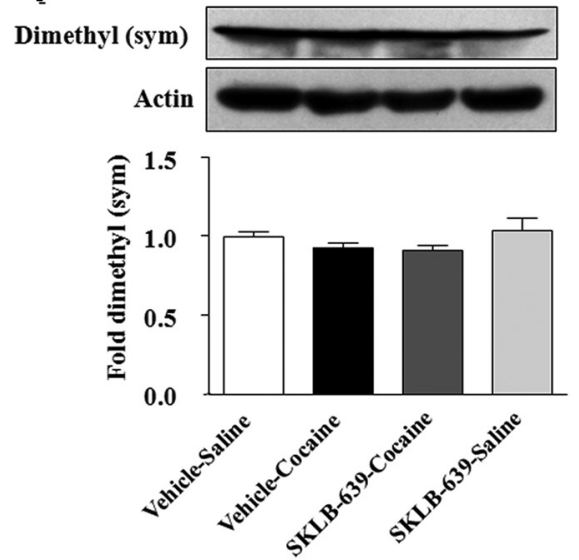

Figure 5. SKLB-639 attenuates cocaine reward via specifically inhibiting modification of $\mathrm{H} 4 \mathrm{R} 3 \mathrm{me} 2 \mathrm{a}$ in vivo. $\boldsymbol{A}$, Timeline of the cocaine CPP experiment. $\boldsymbol{B}$, Intra-NAc SKLB-639 ( $1 \mathrm{~mm}, 1 \mu \mathrm{l} /$ injection) significantly attenuated cocaine CPP. ${ }^{*} p<0.05,{ }^{* * *} p<$ 0.001. n.s., Not significant. $n=13$ Vehicle + Saline, $n=10$ SKLB-639+Saline, $n=11$ Vehicle + Cocaine, $n=13$ SKLB$639+$ Cocaine. C, SKLB-639 decreased expression of H4R3me2a in NAc. ${ }^{*} p<0.05,{ }^{* *} p<0.01$. n.s., Not significant. $n=4$ Vehicle + Saline, $n=4$ Vehicle + Cocaine, $n=4$ SKLB-639+Cocaine, $n=4$ SKLB-639+Saline. D-F, SKLB-639 did not influence levels of H3R2me2a, H3R17me2a, and dimethyl (sym) in NAc in vivo, respectively. $n=4$ Vehicle +Saline, $n=4$ SKLB$639+$ Saline, $n=4$ Vehicle + Cocaine, $n=4$ SKLB-639+Cocaine. Data are presented as the mean \pm SEM.

epigenetic modifying enzyme inhibitors have become available to the chemical biology community (Vedadi et al., 2011). Accumulating evidence shows that selective pharmacological inhibition of catalytic activity in an individual enzyme is a useful strategy to probe its contribution to neuronal dysfunction. However, because of the highly conserved sequence and structure of the catalytic domain within PRMTs, few small-molecule tools are currently available for probing the activity of individual family members. 
A
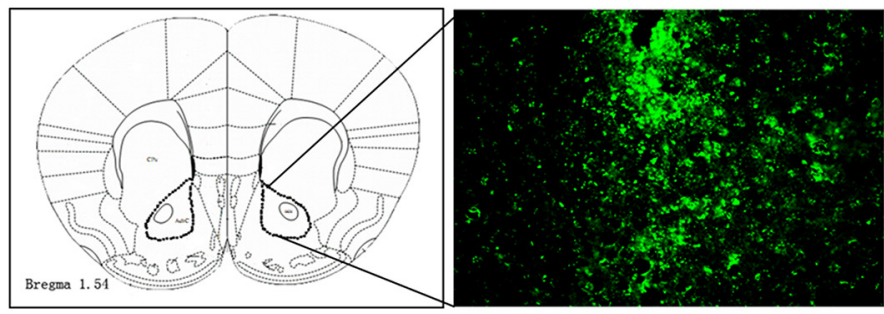

C

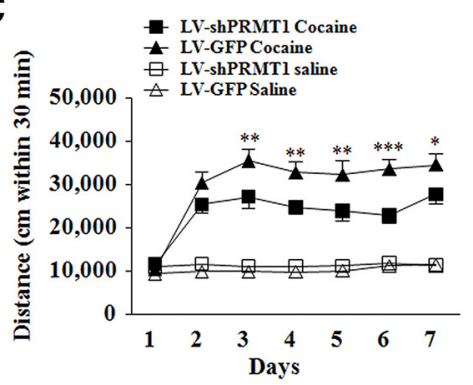

$\mathbf{F}$

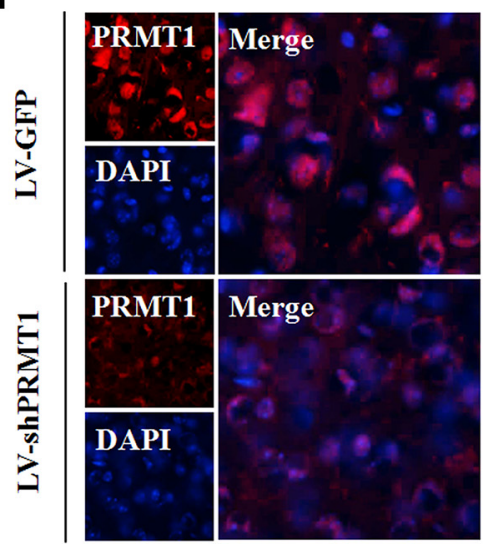

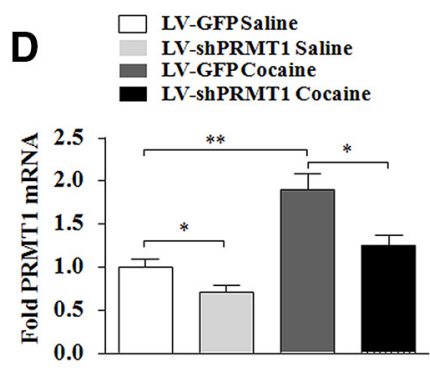

G

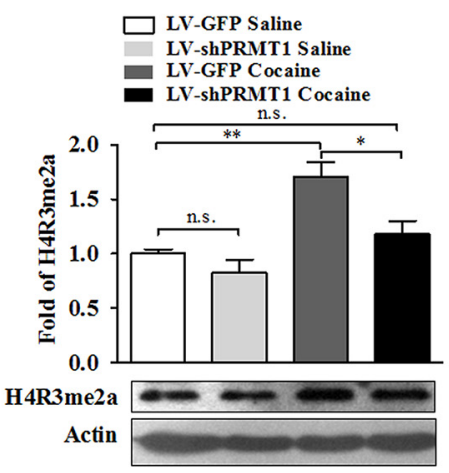

B
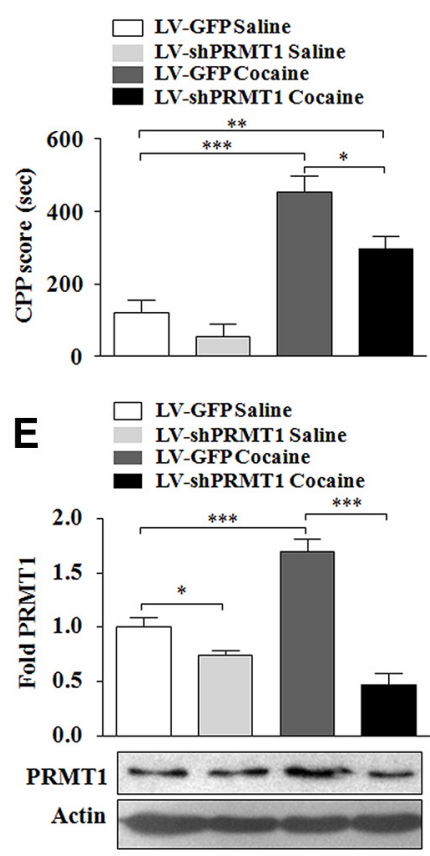

H

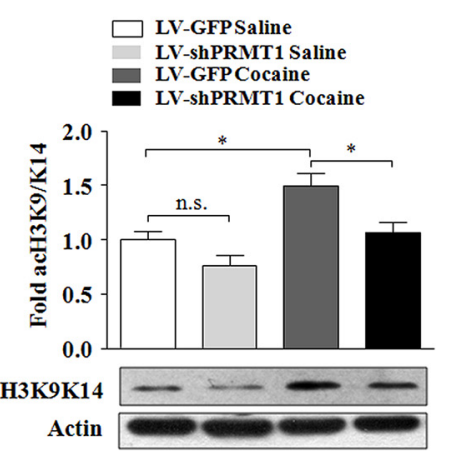

Figure 6. NAc-specific downregulation of PRMT1 regulates cocaine-induced behavioral plasticity. $A$, Verification of anatomical placement and viral infection in NAc; diagram of the coronal brain slice was taken from the mouse brain atlas at $1.54 \mathrm{~mm}$. B, One-way ANOVA revealed that PRMT1 downregulation significantly attenuated the cocaine reward of PRMT1. ${ }^{*} p<0.05,{ }^{* *} p<0.01,{ }^{* * *} p<0.001 . n=13$ LV-GFP Saline, $n=13$ LV-shPRMT1 Saline, $n=11$ LV-GFP Cocaine, $n=12$ LV-shPRMT1 Cocaine. C, LV-shPRMT1 significantly reduced the locomotor activity of mice treated with repeated cocaine injections. $n=11$ LV-GFP Saline, $n=12$ LV-shPRMT1 Saline, $n=12$ LV-GFP Cocaine, $n=13$ LV-shPRMT1 Cocaine. ${ }^{*} p<$ $0.05,{ }^{* *} p<0.01$, and ${ }^{* * *} p<0.001$ compared with LV-shPRMT1 cocaine group. D, PRMT1 mRNA level in NAc of mice infected with LV-shPRMT1 after cocaine CPP. ${ }^{*} p<0.05,{ }^{* *} p<$ $0.01 . n=6$ /condition. $E$, PRMT1 protein level in NAc of mice infected with LV-shPRMT1 after cocaine CPP. ${ }^{*} p<0.05,{ }^{* * *} p<0.001 . n=7$ LV-GFP saline and LV-shPRMT1 saline group, $n=3$ LV-GFP cocaine and LV-shPRMT1 cocaine group. $F$, Representative immunoblots demonstrating the downregulated expression of PRMT1 in NAc of mice with cocaine CPP test. $\boldsymbol{G}$, Protein levels of H4R3me2a in NAc of mice injected with LV-GFP or LV-shPRMT1 after cocaine CPP. ${ }^{*} p<0.05,{ }^{* *} p<0.01$. n.s., Not significant. $n=4 /$ condition. $\boldsymbol{H}$, Protein levels of acH3K9/K14 in NAc of mice infected with LV-GFP or LV-shPRMT1 after cocaine CPP. ${ }^{*} p<0.05$. n.s., Not significant. $n=4 /$ condition. All data are presented as the mean \pm SEM.

A key aspect of this work, starting with a structure-based virtual screening and followed by a chemical optimization approach, is that we identify a novel and structurally unique compound (SKLB-639) that effectively and preferentially inhibits the methylation of H4R3me2a by PRMT1 in vitro and in vivo. Although the PRMTs share key residues, including the Double-E loop in the peptide substrate-binding cavity, the amino acids proximal to the cavity are not identical. Our docking structure suggests that the 5-nitropyrimidine-4,6-diamine scaffold, which enforces strong spatial constraints, may allow for high target selectivity. Although there is some inhibition of PRMT3 and PRMT4 in in vitro experiments, it is quite small compared with that against PRMT1. Our in vivo data suggest that the ability of
SKLB-639, at a dose of $1 \mathrm{~mm}$, can decrease PRMT1 activity in a selective manner, in that we do not observe PRMT4- or PRMT6dependent modifications of H3R17me2a and H3R2me2a; nor do we observe the changes expected to follow from type II PRMT activity in the NAc of mice following administration of intra-NAc SKLB-639 (Guccione et al., 2007; Nicholson et al., 2009; Selvi et al., 2010). While the current compound represents a chemical and biological probe for in vitro and in vivo experiments, we do not suggest that this compound itself could form a basis for patient treatment. Pharmacological optimization and clinical study of SKLB-639 do, however, hold great promise for eventual clinical use.

Histone H4 Arg3 methylation is one of the least well characterized histone tail modifications. PRMT1 produces mono- 
A

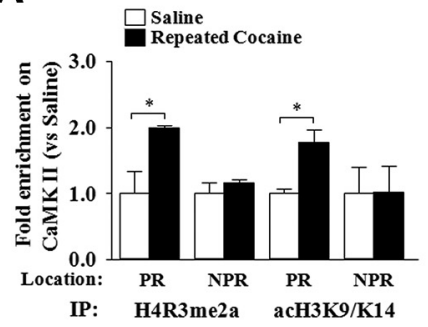

B

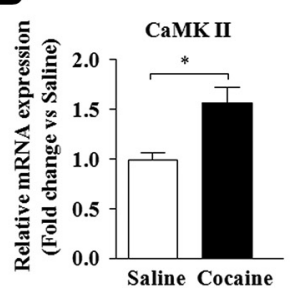

C

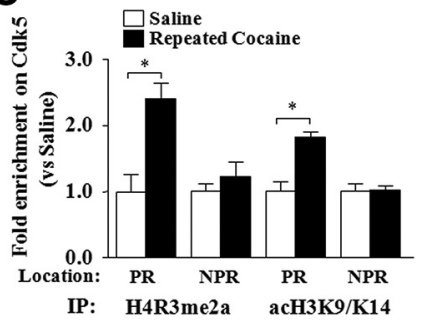

D

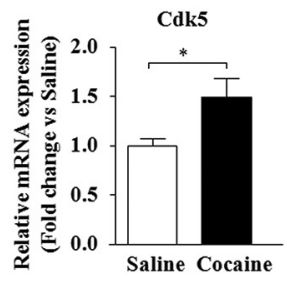

E

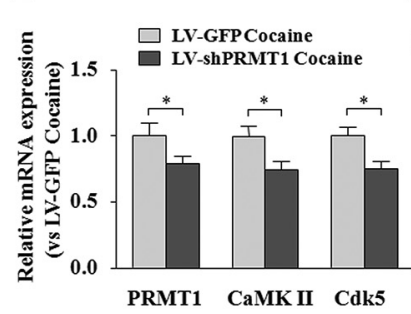

F

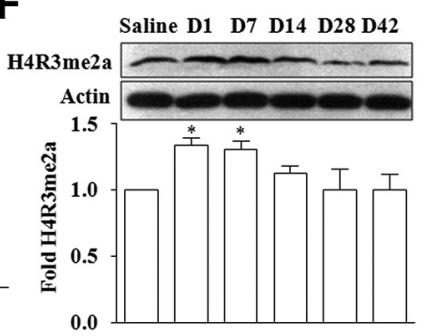

G

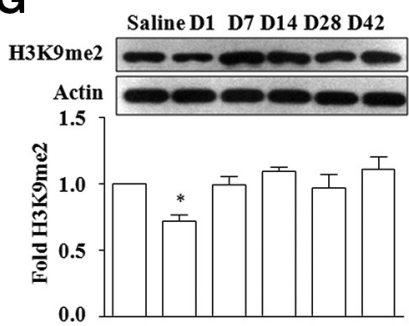

H

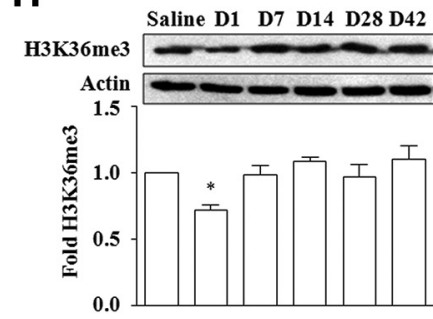

Figure 7. Histone modifications following repeated cocaine injections. A, CaMKIl showed concerted changes in H4R3me2a and acH3K9/K14 enrichment following repeated cocaine injections. PR, promoter region; NPR, nonpromoter region. ${ }^{*} p<0.05 . n=3 /$ condition, $n=4$ animals pooled/condition. $B$, CaMKII was upregulated at the transcriptional level after repeated cocaine injections. ${ }^{*} p<0.05 . n=5 /$ condition. C, Cdk5 promoter obviously increased the binding of H4R3me2 and acH3K9/K14. ${ }^{*} p<0.05, n=3 /$ condition, $n=4$ animals pooled/condition. $D$, Cdk5 was increased in mRNA expression following repeated cocaine challenge. ${ }^{*} p<0.05 . n=5 /$ condition. $E$, With suppression of PRMT1 in NAc, the expression of CaMKII and Cdk5 in the LV-shPRMT1 cocaine group was decreased compared with that of the LV-GFP cocaine group following CPP. ${ }^{*} p<0.05 . n=5 /$ condition. $\boldsymbol{F}-\boldsymbol{H}$, Expressions of H4R3me2a, H3K9me2, and H3K36me3 in NAc after repeated noncontingent cocaine injections withdrawal at day 1 (D1), day 7 (D7), day 14 (D14), day 28 (D28), and day 42 (D42), respectively. Actin was used as the loading control. ${ }^{*} p<0.05$ vs saline, $n=$ 3/condition). Each bar represents the mean \pm SEM.

symmetric and asymmetric dimethyl-arginines, which play an important role in the transcriptional activation and maintenance of an active chromatin environment (Yang et al., 2010; Dhar et al., 2012). In vitro studies have demonstrated that PRMT1 methylates H4 Arg3, and that, once methylated, H4 and $\mathrm{H} 3$ are better substrates for acetyltransferases; however, once acetylated, the histone tails lose their ability to become methylated by PRMT1, suggesting a unidirectional relay of histone-marking processes in a transcription cycle (Wang et al., 2001). Consistent with these previous observations, our data show that PRMT1 knockdown may result in reduced H4R3me2a expression to alter potential H3K9/K14 acetylation by acetyltransferases, and to eventually result in diminished acH3K9/K14 modification in NAc. In the present study, the effect of shRNA-mediated PRMT1 knockdown on H4 Arg3 methylation shows that PRMT1 is the principal enzyme responsible for this modification. Importantly, H4 Arg3 methylation is necessary for lysine acetylation of $\mathrm{H} 3$ in brain reward regions. In fact, histone arginine methylation occurring in conjunction with modifications in lysine methylation, lysine acetylation, and serine phosphorylation strongly suggests functional cross talk among the pathways.

Cocaine-induced behavioral abnormalities are particularly long lived. Thereby, one of the critical challenges in the field is to identify relatively stable drug-induced changes in the brain (Nestler, 2004). Prior investigations have shown that histone lysine acetylation and methylation modifications play crucial roles in gene transcription. In particular, it has been known that $C a M K I I$ and $C d k 5$ are examples of genes that are induced by repeated cocaine administration, and that can individually influence aspects of cocaine addiction. Since H4R3me2a and acH3K9/K14 represent markers that are correlated with increased gene transcription, the enrichment of H4R3me2a and acH3K9/K14, as well as the elevated expression of CaMKII and
Cdk5 mRNA, suggest that these genes are downstream targets of PRMT1 following repeated noncontingent cocaine injection. Dimethylation of arginine is a relatively stable change because it is unclear whether this modification can be enzymatically reversed. At present, Jumonji domain-containing protein 6 and peptidylarginine deiminases are considered two putative arginine demethylases, but they are not "true" demethylases because they cannot remove the methyl group from methylated arginine residues or free arginine from the derivatives (Sarmento et al., 2004; Bedford and Clarke, 2009; Di Lorenzo and Bedford, 2011). Theoretically, arginine dimethylation likely represents more of a "long-term" activation marker representing a change in transcriptional regulation. In fact, our data show that H4R3me2a is not a permanent modification, as previously suggested, since it returns to normal at $14 \mathrm{~d}$ after cocaine withdrawal. However, it is a relatively stable modification compared with H3K36me3 and even H3K9me2, which have been described with cocaine addiction (Maze et al., 2010a). Considering the persistent nature of addiction, altered H4R3me2a within gene promoters, as suggested in the present study, could more persistently influence gene expression in NAc to maintain cocaine-induced CPP.

The molecular mechanisms by which arginine methylation of histones by PRMT1 contributes to chromatin remodeling and transcriptional activation in response to cocaine remain to be determined. Our findings indicate that H4R3me2a affects the transcriptional activity of its target genes following repeated cocaine injections. In addition, the absence of PRMT1 may interfere with the recruitment of histone acetyltransferases due to the absence of methylation on H4 Arg3. Thus, the modification of arginine methylation at histone $\mathrm{H} 4$ sites may represent an integral component of the transcriptional activation process. Together, this work demonstrates the importance of PRMT1 in the modification of H4R3me2a 
and associated cocaine-induced CPP, and further emphasizes the key role of histone modifications in controlling the particularly long-lived features of drug-induced behavioral plasticity.

\section{References}

Bedford MT, Clarke SG (2009) Protein arginine methylation in mammals: who, what, and why. Mol Cell 33:1-13. CrossRef Medline

Belin D, Mar AC, Dalley JW, Robbins TW, Everitt BJ (2008) High impulsivity predicts the switch to compulsive cocaine-taking. Science 320:13521355. CrossRef Medline

Bibb JA, Chen J, Taylor JR, Svenningsson P, Nishi A, Snyder GL, Yan Z, Sagawa ZK, Ouimet CC, Nairn AC, Nestler EJ, Greengard P (2001) Effects of chronic exposure to cocaine are regulated by the neuronal protein Cdk5. Nature 410:376-380. CrossRef Medline

Birkaya B, Aletta JM (2005) NGF promotes copper accumulation required for optimum neurite outgrowth and protein methylation. J Neurobiol 63:49-61. CrossRef Medline

Bissinger EM, Heinke R, Spannhoff A, Eberlin A, Metzger E, Cura V, Hassenboehler P, Cavarelli J, Schüle R, Bedford MT, Sippl W, Jung M (2011) Acyl derivatives of $\mathrm{p}$-aminosulfonamides and dapsone as new inhibitors of the arginine methyltransferase hPRMT1. Bioorg Med Chem 19:37173731. CrossRef Medline

Cheng D, Yadav N, King RW, Swanson MS, Weinstein EJ, Bedford MT (2004) Small molecule regulators of protein arginine methyltransferases. J Biol Chem 279:23892-23899. CrossRef Medline

Cheng D, Valente S, Castellano S, Sbardella G, Di Santo R, Costi R, Bedford MT, Mai A (2011) Novel 3, 5-bis (bromohydroxybenzylidene) piperidin-4-ones as coactivator-associated arginine methyltransferase 1 inhibitors: enzyme selectivity and cellular activity. J Med Chem 54:4928-4932. CrossRef Medline

Deng JV, Rodriguiz RM, Hutchinson AN, Kim IH, Wetsel WC, West AE (2010) MeCP2 in the nucleus accumbens contributes to neural and behavioral responses to psychostimulants. Nat Neurosci 13:1128-1136. CrossRef Medline

Dhar SS, Lee SH, Kan PY, Voigt P, Ma L, Shi X, Reinberg D, Lee MG (2012) Trans-tail regulation of MLL4-catalyzed H3K4 methylation by H4R3 symmetric dimethylation is mediated by a tandem PHD of MLL4. Genes Dev 26:2749-2762. CrossRef Medline

Di Lorenzo A, Bedford MT (2011) Histone arginine methylation. FEBS Lett 585:2024-2031. CrossRef Medline

Guccione E, Bassi C, Casadio F, Martinato F, Cesaroni M, Schuchlautz H, Lüscher B, Amati B (2007) Methylation of histone H3R2 by PRMT6 and H3K4 by an MLL complex are mutually exclusive. Nature 449:933-937. CrossRef Medline

Heinke R, Spannhoff A, Meier R, Trojer P, Bauer I, Jung M, Sippl W (2009) Virtual screening and biological characterization of novel histone arginine methyltransferase PRMT1 inhibitors. ChemMedChem 4:69-77. CrossRef Medline

Hevia H, Varela-Rey M, Corrales FJ, Berasain C, Martínez-Chantar ML, Latasa MU, Lu SC, Mato JM, García-Trevijano ER, Avila MA (2004) 5 '-methylthioadenosine modulates the inflammatory response to endotoxin in mice and in rat hepatocytes. Hepatology 39:1088-1098. CrossRef Medline

Iwasaki H, Yada T (2007) Protein arginine methylation regulates insulin signaling in L6 skeletal muscle cells. Biochem Biophys Res Commun 364: 1015-1021. CrossRef Medline

Kadivar M, Farahmandfar M, Ranjbar FE, Zarrindast MR (2014) Increased calcium/calmodulin-dependent protein kinase II activity by morphinesensitization in rat hippocampus. Behav Brain Res 267:74-82. CrossRef Medline

Knutson SK, Wigle TJ, Warholic NM, Sneeringer CJ, Allain CJ, Klaus CR, Sacks JD, Raimondi A, Majer CR, Song J, Scott MP, Jin L, Smith JJ, Olhava EJ, Chesworth R, Moyer MP, Richon VM, Copeland RA, Keilhack H, Pollock RM, et al. (2012) A selective inhibitor of EZH2 blocks H3K27 methylation and kills mutant lymphoma cells. Nat Chem Biol 8:890-896. CrossRef Medline

Kumar A, Choi KH, Renthal W, Tsankova NM, Theobald DE, Truong HT, Russo SJ, Laplant Q, Sasaki TS, Whistler KN, Self DW, Nestler EJ (2005) Chromatin remodeling is a key mechanism underlying cocaine-induced plasticity in striatum. Neuron 48:303-314. CrossRef Medline

LaPlant Q, Vialou V, Covington HE 3rd, Dumitriu D, Feng J, Warren BL,
Maze I, Dietz DM, Watts EL, Iñiguez SD, Koo JW, Mouzon E, Renthal W, Hollis F, Wang H, Noonan MA, Ren Y, Eisch AJ, Bolaños CA, Kabbaj M, et al. (2010) Dnmt3a regulates emotional behavior and spine plasticity in the nucleus accumbens. Nat Neurosci 13:11371143. CrossRef Medline

Lee YH, Stallcup MR (2009) Minireview: protein arginine methylation of nonhistone proteins in transcriptional regulation. Mol Endocrinol 23: 425-433. CrossRef Medline

Li X, Hu X, Patel B, Zhou Z, Liang S, Ybarra R, Qiu Y, Felsenfeld G, Bungert J, Huang S (2010) H4R3 methylation facilitates $\beta$-globin transcription by regulating histone acetyltransferase binding and $\mathrm{H} 3$ acetylation. Blood 115:2028-2037. CrossRef Medline

Li Y, Hu Z, Chen B, Bu Q, Lu W, Deng Y, Zhu R, Shao X, Hou J, Zhao J, Li H, Zhang B, Huang Y, Lv L, Zhao Y, Cen X (2012) Taurine attenuates methamphetamine-induced autophagy and apoptosis in PC12 cells through mTOR signaling pathway. Toxicol Lett 215:1-7. CrossRef Medline

Limm K, Ott C, Wallner S, Mueller DW, Oefner P, Hellerbrand C, Bosserhoff AK (2013) Deregulation of protein methylation in melanoma. Eur J Cancer 49:1305-1313. CrossRef Medline

Lobera M, Madauss KP, Pohlhaus DT, Wright QG, Trocha M, Schmidt DR, Baloglu E, Trump RP, Head MS, Hofmann GA, Murray-Thompson M, Schwartz B, Chakravorty S, Wu Z, Mander PK, Kruidenier L, Reid RA, Burkhart W, Turunen BJ, Rong JX, et al. (2013) Selective class IIa histone deacetylase inhibition via a nonchelating zinc-binding group. Nat Chem Biol 9:319-325. CrossRef Medline

Maze I, Covington HE 3rd, Dietz DM, LaPlant Q, Renthal W, Russo SJ, Mechanic M, Mouzon E, Neve RL, Haggarty SJ, Ren Y, Sampath SC, Hurd YL, Greengard P, Tarakhovsky A, Schaefer A, Nestler EJ (2010a) Essential role of the histone methyltransferase G9a in cocaine-induced plasticity. Science 327:213-216. CrossRef Medline

Maze I, Covington III HE, Dietz DM, LaPlant Q, Renthal W, Russo SJ, Mechanic M, Mouzon E, Neve RL, Haggarty SJ (2010b) Essential role of the histone methyltransferase G9a in cocaine-induced plasticity. Sci STKE 327:213.

Miyata S, Mori Y, Tohyama M (2008) PRMT1 and Btg2 regulates neurite outgrowth of Neuro2a cells. Neurosci Lett 445:162-165. CrossRef Medline

Nestler EJ (2004) Historical review: molecular and cellular mechanisms of opiate and cocaine addiction. Trends Pharmacol Sci 25:210-218. CrossRef Medline

Nicholson TB, Chen T, Richard S (2009) The physiological and pathophysiological role of PRMT1-mediated protein arginine methylation. Pharmacol Res 60:466-474. CrossRef Medline

Noonan MA, Choi KH, Self DW, Eisch AJ (2008) Withdrawal from cocaine self-administration normalizes deficits in proliferation and enhances maturity of adult-generated hippocampal neurons. J Neurosci 28:25162526. CrossRef Medline

Pal S, Sif S (2007) Interplay between chromatin remodelers and protein arginine methyltransferases. J Cell Physiol 213:306-315. CrossRef Medline

Pritchard LM, Hensleigh E, Lynch S (2012) Altered locomotor and stereotyped responses to acute methamphetamine in adolescent, maternally separated rats. Psychopharmacology 223:27-35. CrossRef Medline

Renthal W, Maze I, Krishnan V, Covington HE 3rd, Xiao G, Kumar A, Russo SJ, Graham A, Tsankova N, Kippin TE, Kerstetter KA, Neve RL, Haggarty SJ, McKinsey TA, Bassel-Duby R, Olson EN, Nestler EJ (2007) Histone deacetylase 5 epigenetically controls behavioral adaptations to chronic emotional stimuli. Neuron 56:517-529. CrossRef Medline

Sakamaki J, Daitoku H, Ueno K, Hagiwara A, Yamagata K, Fukamizu A (2011) Arginine methylation of BCL-2 antagonist of cell death (BAD) counteracts its phosphorylation and inactivation by Akt. Proc Natl Acad Sci U S A 108:6085-6090. CrossRef Medline

Sarmento OF, Digilio LC, Wang Y, Perlin J, Herr JC, Allis CD, Coonrod SA (2004) Dynamic alterations of specific histone modifications during early murine development. J Cell Sci 117:4449-4459. CrossRef Medline

Selvi BR, Batta K, Kishore AH, Mantelingu K, Varier RA, Balasubramanyam K, Pradhan SK, Dasgupta D, Sriram S, Agrawal S, Kundu TK (2010) Identification of a novel inhibitor of coactivator-associated arginine methyltransferase 1 (CARM1)-mediated methylation of histone H3 Arg17. J Biol Chem 285:7143-7152. CrossRef Medline

Valjent E, Corbillé AG, Bertran-Gonzalez J, Hervé D, Girault JA (2006) Inhibition of ERK pathway or protein synthesis during reexposure to drugs 
of abuse erases previously learned place preference. Proc Natl Acad Sci U S A 103:2932-2937. CrossRef Medline

Vedadi M, Barsyte-Lovejoy D, Liu F, Rival-Gervier S, Allali-Hassani A, Labrie V, Wigle TJ, Dimaggio PA, Wasney GA, Siarheyeva A, Dong A, Tempel W, Wang SC, Chen X, Chau I, Mangano TJ, Huang XP, Simpson CD, Pattenden SG, Norris JL, et al. (2011) A chemical probe selectively inhibits G9a and GLP methyltransferase activity in cells. Nat Chem Biol 7:566574. CrossRef Medline

Wang H, Huang Z-Q, Xia L, Feng Q, Erdjument-Bromage H, Strahl BD, Briggs SD, Allis CD, Wong J, Tempst P (2001) Methylation of histone $\mathrm{H} 4$ at arginine 3 facilitating transcriptional activation by nuclear hormone receptor. Sci Signal 293:853.

Wang J, Chen L, Sinha SH, Liang Z, Chai H, Muniyan S, Chou YW, Yang C, Yan L, Feng Y, Li KK, Lin MF, Jiang H, Zheng YG, Luo C (2012a) Pharmacophore-based virtual screening and biological evaluation of small molecule inhibitors for protein arginine methylation. J Med Chem 55:7978-7987. CrossRef Medline

Wang S, Tan X, Yang B, Yin B, Yuan J, Qiang B, Peng XZ (2012b) The role of protein arginine-methyltransferase 1 in gliomagenesis. BMB Rep 45: 470-475. CrossRef Medline

Yan L, Yan C, Qian K, Su H, Kofsky-Wofford S, Lee WC, Zhao X, Ho MC, Ivanov I, Zheng YG (2014) Diamidine compounds for selective inhibition of protein arginine methyltransferase 1. J Med Chem 57:2611-2622. CrossRef Medline

Yang Y, Lu Y, Espejo A, Wu J, Xu W, Liang S, Bedford MT (2010) TDRD3 is an effector molecule for arginine-methylated histone marks. Mol Cell 40:1016-1023. CrossRef Medline

Zhang X, Cheng X (2003) Structure of the predominant protein arginine methyltransferase PRMT1 and analysis of its binding to substrate peptides. Structure 11:509-520. CrossRef Medline 